\title{
SECOND-ORDER STRUCTURED DEFORMATIONS: RELAXATION, INTEGRAL REPRESENTATION AND APPLICATIONS
}

\author{
ANA CRISTINA BARROSO, JOSÉ MATIAS, MARCO MORANDOTTI, AND DAVID R.OWEN
}

\begin{abstract}
Second-order structured deformations of continua provide an extension of the multiscale geometry of first-order structured deformations by taking into account the effects of submacroscopic bending and curving. We derive here an integral representation for a relaxed energy functional in the setting of second-order structured deformations. Our derivation covers inhomogeneous initial energy densities (i.e., with explicit dependence on the position); finally, we provide explicit formulas for bulk relaxed energies as well as anticipated applications.
\end{abstract}

\section{INTRODUCTION}

A first-order structured deformation $(g, G)$ from a region $\Omega \subset \mathbb{R}^{N}$ provides not only a macroscopic deformation field $g: \Omega \rightarrow \mathbb{R}^{d}$ but also a field $G: \Omega \rightarrow \mathbb{R}^{d \times N}$ intended to capture the contributions at the macrolevel of smooth submacroscopic geometrical changes such as stretching, shearing, and rotation. Indeed, in a variety of settings [7, 12, 15, 32], one can prove an approximation theorem to the effect that there exist a sequence of mappings $u_{n}: \Omega \rightarrow \mathbb{R}^{d}$ that converges to $g$ and whose gradients $\nabla u_{n}: \Omega \rightarrow \mathbb{R}^{d \times N}$ converge to $G$. In addition, one obtains a formula that identifies the difference $M:=\nabla g-G=\nabla \lim _{n \rightarrow \infty} u_{n}-\lim _{n \rightarrow \infty} \nabla u_{n}$ as a limit of "disarrangements", i.e., of averages of directed jumps $\left[u_{n}\right] \otimes \nu_{u_{n}}$ in the approximating mappings (here, $\nu_{u_{n}}$ denotes the normal to the jump-set of $u_{n}$ ). These disarrangements include the formation of voids, slips, and separations occuring at submacroscopic levels. $M$ is called the (volume) density of disarrangements, and, because $G=\lim _{n \rightarrow \infty} \nabla u_{n}$ does not reflect the jumps in $u_{n}$, the field $G$ is called the deformation without disarrangements.

The additive decomposition $\nabla g=G+M$ along with the identifications above of $G$ and $M$ provides a richer geometrical setting in which to study mechanisms for storing mechanical energy. The main approach to assigning an energy to a continuum undergoing structured deformations $(g, G)$ is to assume that such an assignment $E\left(u_{n}\right)$ is available for the approximating deformations $u_{n}$ in the form of a bulk energy plus an interfacial energy, $E_{B}\left(u_{n}\right)+E_{I}\left(u_{n}\right)$, and to assign to $(g, G)$ the relaxed energy

$$
E(g, G):=\inf _{\left\{u_{n}\right\}}\left\{\liminf _{n \rightarrow \infty}\left(E_{B}\left(u_{n}\right)+E_{I}\left(u_{n}\right)\right): u_{n} \rightarrow g, \nabla u_{n} \rightarrow G\right\}
$$

where the class of approximating functions and the two senses of convergence are to be specified in such a way that an appropriate version of the approximation theorem can be verified. This approach was first studied in [12], where additive decompositions

$$
E(g, G)=E_{\text {bulk }}(g, G)+E_{\text {int }}(g, G)
$$

of the relaxed energies as well as a variety of properties of the associated bulk and interfacial energy densities were established. In a different setting, the study [7] used similar techniques to obtain an additive decomposition of this form along with the additional decomposition $E_{\text {bulk }}(g, G)=E_{\text {bulk }}^{1}(M)+E_{\text {bulk }}^{2}(G, \nabla g)$. See the survey article [6] for details and comparisons. The article [25] addresses issues related to additional decomposition of $E_{\text {bulk }}$ in [7], while [14] obtains detailed information about relaxed energies in the case of one-dimensional structured deformations.

Date: November 21, 2018. Preprint SISSA: 37/MATE/2016.

2010 Mathematics Subject Classification. 49J45 (74G65, 74M25, 15A99) . 
The various studies of relaxed energies in the case of first-order structured deformations $(g, G)$ cited above do not account explicitly for the contributions to the energy of "gradient disarrangements", i.e., of jumps in $\nabla u_{n}$, with $u_{n}$ converging to $g$ and $\nabla u_{n}$ converging to $G$. The multiscale geometry of structured deformations was broadened [28, 30], to provide additional fields capable of describing effects at the macrolevel of gradient disarrangements. A secondorder structured deformation is a triple $(g, G, \Gamma)$ in which $(g, G)$ is a first-order structured deformation (with additional smoothness granted to $g$ and $G$ ) and $\Gamma: \Omega \rightarrow \mathbb{R}^{d \times N \times N}$ is a field intended to describe the contributions at the macrolevel of smooth bending and of curving at submacroscopic levels. In [28, 30], various versions of approximation theorems are obtained that provide sequences of approximations $u_{n}$ with $u_{n}$ converging to $g, \nabla u_{n}$ converging to $G$, and $\nabla^{2} u_{n}$ converging to $\Gamma$. The decomposition $\nabla g=G+M$ remains valid here and implies the higher-order decomposition

$$
\nabla^{2} g=\nabla M+(\nabla G-\Gamma)+\Gamma
$$

In view of the approximation theorem, we can write

$$
\nabla G-\Gamma=\nabla \lim _{n \rightarrow \infty} \nabla u_{n}-\lim _{n \rightarrow \infty} \nabla^{2} u_{n} .
$$

As a consequence, $\nabla G-\Gamma$ can be shown to be a limit of averages of directed jumps $\left[\nabla u_{n}\right] \otimes \nu_{\nabla u_{n}}$ in analogy with the corresponding result for $\nabla g-G$, so that $\nabla G-\Gamma$ emerges as a density of gradient disarrangements.

In this article, we use this background to study the relaxation of energies in a specific mathematical setting for second-order structured deformations $(g, G, \Gamma)$, the so-called " $S B V^{2}$ setting", see [16]. The results in [12] and [7] for the energetics of first-order structured deformations and those of [30] provide a guide for our analysis of energetics in the second-order case. Beyond providing an analysis in the second-order case, we broaden the scope further by following ideas in [9] in order to include in our analysis the case of "inhomogeneous energetic response", i.e., the case in which initial bulk and interfacial densities can depend explicitly on location in the body.

The overall plan of this work in the ensuing sections is as follows. In Section 2 we fix the notation and recall some auxiliary results used throughout the paper. The problem, our hypotheses and the main result, Theorem 3.2, are presented in Section 3 . In Section 4 we prove some preliminary results and, in particular, show that our energy functional can be decomposed into a sum of two lower order functionals. Section 5 is devoted to the proof of Theorem 3.2, and finally, in Section 6, we give an example in which the formula in Theorem 3.2 for the bulk relaxed energy density can be calculated explicitly, thus providing an explicit formula in terms of $\nabla G-\Gamma$ for the volume density of the non-tangential part of jumps in directional derivatives of approximations. We further indicate in Section 6 applications of the energetics of second-order structured deformations in the study of elastic bodies undergoing disarrangements.

\section{Preliminaries}

The purpose of this section is to give a brief overview of the concepts and results that are used in the sequel. Almost all these results are stated without proofs as they can be readily found in the references given below.

2.1. Notation. Throughout the text $\Omega \subset \mathbb{R}^{N}, N \geqslant 1$, will denote an open bounded set and we will use the following notations:

- $\mathcal{O}(\Omega)$ is the family of all open subsets of $\Omega$,

- $\mathcal{M}(\Omega)$ is the set of finite Radon measures on $\Omega$,

- $\mathcal{M}^{+}(\Omega)$ is the set of finite and positive Radon measures on $\Omega$,

- $\|\mu\|$ stands for the total variation of a measure $\mu \in \mathcal{M}(\Omega)$,

- $S^{N-1}$ stands for the unit sphere in $\mathbb{R}^{N}$,

- $e_{i}$ denotes the $i^{\text {th }}$ element of the canonical basis of $\mathbb{R}^{N}$, for $i=1, \ldots, N$.

- $Q$ denotes the unit cube centered at the origin with faces orthogonal to the coordinate axes, 
- $Q(x, \delta)$ denotes a cube centered at $x \in \Omega$ with side length $\delta$ and with two of its faces orthogonal to $e_{N}$,

- $Q_{\nu}(x, \delta)$ is a cube centered at $x \in \Omega$ with side length $\delta$ and with two of its faces orthogonal to $\nu \in S^{N-1}$,

- $Q_{\nu}:=Q_{\nu}(0,1)$,

- $C$ represents a generic constant whose value might change from line to line,

- $\lim _{n, m \rightarrow+\infty}:=\lim _{n \rightarrow+\infty} \lim _{m \rightarrow+\infty}$ while $\lim _{m, n \rightarrow+\infty}:=\lim _{m \rightarrow+\infty} \lim _{n \rightarrow+\infty}$,

2.2. Measure Theory. We recall Reshetnyak's Theorem on weak convergence of vector measures (see Reshetnyak [31]; see also Ambrosio, Fusco and Pallara [4]).

Theorem 2.1. Let $\mu, \mu_{n}$ be $\mathbb{R}^{d}$-valued finite Radon measures in $\Omega$ such that $\mu_{n} \stackrel{*}{\rightarrow} \mu$ in $\Omega$ and such that $\left\|\mu_{n}\right\|(\Omega) \rightarrow\|\mu\|(\Omega)$. Then

$$
\lim _{n \rightarrow+\infty} \int_{\Omega} f\left(x, \frac{\mu_{n}}{\left\|\mu_{n}\right\|}(x)\right) d\left\|\mu_{n}\right\|(x)=\int_{\Omega} f\left(x, \frac{\mu}{\|\mu\|}(x)\right) d\|\mu\|(x)
$$

for every continuous and bounded function $f: \Omega \times S^{d-1} \rightarrow \mathbb{R}$.

2.3. BV Functions. In this section we briefly summarize some facts on functions of bounded variation that will be used throughout the paper. We refer to [4, 21, 22, 23, 33] for a detailed description of this subject.

A function $u \in L^{1}\left(\Omega ; \mathbb{R}^{d}\right)$ is said to be of bounded variation, and we write $u \in B V\left(\Omega ; \mathbb{R}^{d}\right)$, if all its first order distributional derivatives $D_{j} u_{i} \in \mathcal{M}(\Omega)$ for $i=1, \ldots, d$ and $j=1, \ldots, N$. The matrix-valued measure whose entries are $D_{j} u_{i}$ is denoted by $D u$. By the Lebesgue Decomposition Theorem $D u$ can be split into the sum of two mutually singular measures $D^{a} u$ and $D^{s} u$ (the absolutely continuous part and the singular part, respectively, of $D u$ with respect to the Lebesgue measure $\mathcal{L}^{N}$ ). By $\nabla u$ we denote the Radon-Nikodým derivative of $D^{a} u$ with respect to $\mathcal{L}^{N}$, so that we can write

$$
D u=\nabla u \mathcal{L}^{N}\left\lfloor\Omega+D^{s} u .\right.
$$

Let $\Omega_{u}$ be the set of points where the approximate limit of $u$ exists, i.e., points $x \in \Omega$ for which there exists $z \in \mathbb{R}^{N}$ such that

$$
\lim _{\varepsilon \rightarrow 0^{+}} f_{Q(x, \varepsilon)}|u(y)-z| d y=0 .
$$

If $x \in \Omega_{u}$ and $z=u(x)$ we say that $u$ is approximately continuous at $x$ (or that $x$ is a Lebesgue point of $u$ ). The function $u$ is approximately continuous for $\mathcal{L}^{N}$-a.e. $x \in \Omega_{u}$.

The jump set of the function $u$, denoted by $S_{u}$, is the set of points $x \in \Omega \backslash \Omega_{u}$ for which there exist $a, b \in \mathbb{R}^{d}$ and a unit vector $\nu \in S^{N-1}$, normal to $S_{u}$ at $x$, such that $a \neq b$ and

$$
\lim _{\varepsilon \rightarrow 0^{+}} \frac{1}{\varepsilon^{N}} \int_{\left\{y \in Q_{\nu}(x, \varepsilon):(y-x) \cdot \nu>0\right\}}|u(y)-a| d y=0, \quad \lim _{\varepsilon \rightarrow 0^{+}} \frac{1}{\varepsilon^{N}} \int_{\left\{y \in Q_{\nu}(x, \varepsilon):(y-x) \cdot \nu<0\right\}}|u(y)-b| d y=0 .
$$

The triple $(a, b, \nu)$ is uniquely determined by the conditions above up to a permutation of $(a, b)$ and a change of sign of $\nu$ and is denoted by $\left(u^{+}(x), u^{-}(x), \nu_{u}(x)\right)$.

If $u \in B V(\Omega)$ it is well known that $S_{u}$ is countably $(N-1)$-rectifiable, see [4], and the following decomposition holds

$$
D u=\nabla u \mathcal{L}^{N}\left\lfloor\Omega+[u] \otimes \nu_{u} \mathcal{H}^{N-1}\left\lfloor S_{u}+D^{c} u,\right.\right.
$$

where $[u]:=u^{+}-u^{-}$and $D^{c} u$ is the Cantor part of the measure $D u$.

Throughout this paper we shall employ for convenience the slightly abusive notation $[f(x)]$ in place of the more accurate notation $[f](x)$ for the difference $f^{+}(x)-f^{-}(x)$.

We also recall that a measurable subset $E \subset \mathbb{R}^{N}$ is a set of finite perimeter in $\Omega$ if the characteristic function $\chi_{E}$ of $E$ is a function of bounded variation. In this case, the perimeter of $E$ in $\Omega$ is given by the total variation of $\chi_{E}$ in $\Omega$, i.e., $\operatorname{Per}_{\Omega}(E):=\left|D \chi_{E}\right|(\Omega)$.

The following theorem is a variant of a well-known approximation result for sets of finite perimeter and it will be used in the proof of the upper bound inequalities in Proposition 5.6 and Theorem 5.7 . 
Theorem 2.2 ([8, Lemma 3.1]). Let $\Omega$ be an open, bounded set with Lipschitz boundary and let $E$ be a subset of $\Omega$ with $\operatorname{Per}_{\Omega}(E)<+\infty$. There exists a sequence $\left\{E_{n}\right\}$ of polyhedral sets (i.e., for each $n, E_{n}$ is a bounded Lipschitz domain with $\partial E_{n}=H_{1, n} \cup H_{2, n} \cup \ldots H_{L_{n}, n}$, where each $H_{j, n}$ is a closed subset of a hyperplane $\left\{x \in \mathbb{R}^{N}: x \cdot \nu_{j}=c_{j}\right\}$, for some $c_{j} \in \mathbb{R}$ and $\nu_{j} \in S^{N-1}$, $\left.j=1, \ldots, L_{n}, L_{n} \in \mathbb{N}\right)$ satisfying the following properties:

(i) $\chi_{E_{n}} \rightarrow \chi_{E}$ in $L^{1}(\Omega)$, as $n \rightarrow+\infty$,

(ii) $\lim _{n \rightarrow+\infty} \operatorname{Per}_{\Omega}\left(E_{n}\right)=\operatorname{Per}_{\Omega}(E)$,

(iii) $\mathcal{H}^{N-1}\left(\partial^{*} E_{n} \cap \partial \Omega\right)=0\left(\partial^{*} E\right.$ being the reduced boundary of $E$, see [4]),

(iv) $\mathcal{L}^{N}\left(E_{n}\right)=\mathcal{L}^{N}(E)$.

If $\Omega$ is an open and bounded set with Lipschitz boundary then the outer unit normal to $\partial \Omega$ (denoted by $\nu$ ) exists $\mathcal{H}^{N-1}$-a.e. and the trace for functions in $B V\left(\Omega ; \mathbb{R}^{d}\right)$ is defined.

Lemma 2.3. Let $u \in B V\left(\Omega ; \mathbb{R}^{d}\right)$. There exist piecewise constant functions $u_{n}$ such that $u_{n} \rightarrow u$ in $L^{1}\left(\Omega ; \mathbb{R}^{d}\right)$ and

$$
\|D u\|(\Omega)=\lim _{n \rightarrow+\infty}\left\|D u_{n}\right\|(\Omega)=\lim _{n \rightarrow+\infty} \int_{S_{u_{n}}}\left|\left[u_{n}\right](x)\right| d H^{N-1}(x) .
$$

The space of special functions of bounded variation $S B V\left(\Omega ; \mathbb{R}^{d}\right)$, introduced in [13] to study free discontinuity problems, is the space of functions $u \in B V\left(\Omega ; \mathbb{R}^{d}\right)$ such that $D^{c} u=0$, i.e. for which

$$
D u=\nabla u \mathcal{L}^{N}+[u] \otimes \nu_{u} \mathcal{H}^{N-1}\left\lfloor S_{u} .\right.
$$

The next result is a Lusin-type theorem for gradients due to Alberti [3], and is essential for our arguments.

Theorem 2.4. Let $f \in L^{1}\left(\Omega ; \mathbb{R}^{d \times N}\right)$. There exists $u \in S B V\left(\Omega ; \mathbb{R}^{d}\right)$ and a Borel function $g$ : $\Omega \rightarrow \mathbb{R}^{d \times N}$ such that

$$
\begin{gathered}
D u=f \mathcal{L}^{N}+g \mathcal{H}^{N-1}\left\lfloor S_{u},\right. \\
\int_{S_{u}}|g| d \mathcal{H}^{N-1} \leqslant C\|f\|_{L^{1}\left(\Omega ; \mathbb{R}^{d \times N}\right)} .
\end{gathered}
$$

Moreover,

$$
\|u\|_{L^{1}(\Omega)} \leqslant C\|f\|_{L^{1}\left(\Omega ; \mathbb{R}^{d \times N}\right)} .
$$

The following technical result is a simplified version of Lemma 4.3 in [26].

Lemma 2.5. Let $\Omega \subset \mathbb{R}^{N}$ be open and bounded and let $A \in \mathbb{R}^{d \times N}$. Then there exists $u \in$ $S B V\left(\Omega ; \mathbb{R}^{d}\right)$ such that $\left.u\right|_{\partial \Omega}=0$ and $\nabla u=A$ a.e in $\Omega$. In addition

$$
\left\|D^{s} u\right\|(\Omega) \leqslant C(N)|A||\Omega| .
$$

Following [10, 11], we define

$$
S B V^{2}\left(\Omega ; \mathbb{R}^{d}\right):=\left\{v \in S B V\left(\Omega ; \mathbb{R}^{d}\right): \nabla v \in S B V\left(\Omega ; \mathbb{R}^{d \times N}\right)\right\} .
$$

If $u \in S B V^{2}\left(\Omega ; \mathbb{R}^{d}\right)$ we use the notation $\nabla^{2} u=\nabla(\nabla u)$ to denote the absolutely continuous part of $D(\nabla u)$ with respect to the Lebesgue measure. Analogously, we let

$$
B V^{2}\left(\Omega ; \mathbb{R}^{d}\right)=\left\{v \in B V\left(\Omega ; \mathbb{R}^{d}\right): \nabla v \in B V\left(\Omega ; \mathbb{R}^{d \times N}\right)\right\} .
$$

\section{STATEMENT OF THE PROBLEM AND MAIN RESULT}

We define a second order structured deformation as a triplet

$$
(g, G, \Gamma) \in S B V^{2}\left(\Omega ; \mathbb{R}^{d}\right) \times S B V\left(\Omega ; \mathbb{R}^{d \times N}\right) \times L^{1}\left(\Omega ; \mathbb{R}^{d \times N \times N}\right) .
$$

The set of second order structured deformations will be denoted in the sequel by $S D^{2}\left(\Omega ; \mathbb{R}^{d}\right)$.

Given a function $u \in S B V^{2}\left(\Omega ; \mathbb{R}^{d}\right)$, consider the energy defined by

$$
\begin{aligned}
E(u):=\int_{\Omega} W\left(x, \nabla u(x), \nabla^{2} u(x)\right) d x & +\int_{S_{u}} \Psi_{1}\left(x,[u(x)], \nu_{u}(x)\right) d \mathcal{H}^{N-1}(x) \\
& +\int_{S_{\nabla u}} \Psi_{2}\left(x,[\nabla u(x)], \nu_{\nabla u}(x)\right) d \mathcal{H}^{N-1}(x),
\end{aligned}
$$


where the densities $W: \Omega \times \mathbb{R}^{d \times N} \times \mathbb{R}^{d \times N \times N} \rightarrow\left[0,+\infty\left[, \Psi_{1}: \Omega \times \mathbb{R}^{d} \times S^{N-1} \rightarrow[0,+\infty[\right.\right.$ and $\Psi_{2}: \Omega \times \mathbb{R}^{d \times N} \times S^{N-1} \rightarrow[0,+\infty[$ satisfy the following hypotheses:

(H1) there exists $C>0$ such that

$$
\frac{1}{C}(|A|+|M|)-C \leqslant W(x, A, M) \leqslant C(1+|A|+|M|)
$$

for all $x \in \Omega, A \in \mathbb{R}^{d \times N}$ and $M \in \mathbb{R}^{d \times N \times N}$;

(H2) there exists $C>0$ such that

$$
\left|W\left(x, A_{1}, M_{1}\right)-W\left(x, A_{2}, M_{2}\right)\right| \leqslant C\left(\left|A_{1}-A_{2}\right|+\left|M_{1}-M_{2}\right|\right)
$$

for all $x \in \Omega, A_{i} \in \mathbb{R}^{d \times N}$ and $M_{i} \in \mathbb{R}^{d \times N \times N}, i=1,2$;

(H3) for every $x_{0} \in \Omega$ and for every $\varepsilon>0$ there exists a $\delta>0$ such that

$$
\left|x-x_{0}\right|<\delta \Rightarrow\left|W(x, A, M)-W\left(x_{0}, A, M\right)\right| \leqslant \varepsilon C(1+|A|+|M|),
$$

for all $x \in \Omega, A \in \mathbb{R}^{d \times N}$ and $M \in \mathbb{R}^{d \times N \times N}$;

(H4) there exist $0<\alpha<1$ and $L>0$ such that

$$
\left|W^{\infty}(x, A, M)-\frac{W(x, A, t M)}{t}\right| \leqslant \frac{C}{t^{\alpha}}
$$

for all $t>L, x \in \Omega, A \in \mathbb{R}^{d \times N}, M \in \mathbb{R}^{d \times N \times N}$ with $|M|=1$, where $W^{\infty}$ denotes the recession function of $W$ in the variable $M$, i.e.,

$$
W^{\infty}(x, A, M)=\limsup _{t \rightarrow+\infty} \frac{W(x, A, t M)}{t} ;
$$

(H5) there exist $c_{1}>0, K_{1}>0$, such that

$$
c_{1}|\lambda| \leqslant \Psi_{1}(x, \lambda, \nu) \leqslant K_{1}|\lambda|,
$$

for all $x \in \Omega, \lambda \in \mathbb{R}^{d}$ and $\nu \in S^{N-1}$;

there exist $c_{2}>0, K_{2}>0$, such that

$$
c_{2}|\Lambda| \leqslant \Psi_{2}(x, \Lambda, \nu) \leqslant K_{2}|\Lambda|,
$$

for all $x \in \Omega, \Lambda \in \mathbb{R}^{d \times N}$ and $\nu \in S^{N-1}$;

(H6) for every $x_{0} \in \Omega$ and for every $\varepsilon>0$ there exist $\delta>0$ and $C_{1}, C_{2}>0$ such that

$$
\begin{aligned}
& \left|x-x_{0}\right|<\delta \Rightarrow\left|\Psi_{1}\left(x_{0}, \lambda, \nu\right)-\Psi_{1}(x, \lambda, \nu)\right| \leqslant \varepsilon C_{1}|\lambda|, \\
& \left|x-x_{0}\right|<\delta \Rightarrow\left|\Psi_{2}\left(x_{0}, \Lambda, \nu\right)-\Psi_{2}(x, \Lambda, \nu)\right| \leqslant \varepsilon C_{2}|\Lambda|
\end{aligned}
$$

for all $\lambda \in \mathbb{R}^{d}, \Lambda \in \mathbb{R}^{d \times N}$ and $\nu \in S^{N-1}$

(H7) (homogeneity of degree one)

$$
\Psi_{1}(x, t \lambda, \nu)=t \Psi_{1}(x, \lambda, \nu), \quad \Psi_{2}(x, t \Lambda, \nu)=t \Psi_{2}(x, \Lambda, \nu),
$$

for all $x \in \Omega, \nu \in S^{N-1}, \lambda \in \mathbb{R}^{N}, \Lambda \in \mathbb{R}^{d \times N}$ and $t>0$;

(H8) (sub-additivity)

$$
\begin{array}{r}
\Psi_{1}\left(x, \lambda_{1}+\lambda_{2}, \nu\right) \leqslant \Psi_{1}\left(x, \lambda_{1}, \nu\right)+\Psi_{1}\left(x, \lambda_{2}, \nu\right), \\
\Psi_{2}\left(x, \Lambda_{1}+\Lambda_{2}, \nu\right) \leqslant \Psi_{2}\left(x, \Lambda_{1}, \nu\right)+\Psi_{2}\left(x, \Lambda_{2}, \nu\right), \\
\text { for all } x \in \Omega, \nu \in S^{N-1}, \lambda_{i} \in \mathbb{R}^{d}, \Lambda_{i} \in \mathbb{R}^{d \times N}, i=1,2 .
\end{array}
$$

Remark 3.1. (1) We extend $\Psi_{i}, i=1,2$ as homogeneous functions of degree one in the third variable to all of $\mathbb{R}^{N}$.

(2) The hypotheses listed above are similar to the ones in [12] and [7] where there is no explicit dependence on $x$, and with the hypotheses in [9] where the density functions depended explicitly on the variable $x$.

(3) It is well known that the bulk energy may have potential wells and for this reason it is desirable to consider

$$
0 \leqslant W(x, A, M) \leqslant C(1+|A|+|M|),
$$

instead of (H1). However, following the same arguments as in [12], the coercivity assumption can be removed. 
(4) In the case of no explicit dependence on the position variable $x$, the coercivity hypothesis on the interfacial energy densities can be replaced by the extra condition that admissible sequences are bounded in $B V^{2}$-norm. This standard modification of our model covers the case of the example in Section 6 .

(5) It follows immediately from the definition of the recession function and from hypotheses (H1), (H2) and (H3) that there exists $C>0$ such that for all $x \in \Omega, A_{i} \in \mathbb{R}^{d \times N}$ and $M_{i} \in \mathbb{R}^{d \times N \times N}, i=1,2$

$$
\begin{gathered}
\frac{1}{C}\left|M_{1}\right| \leqslant W^{\infty}\left(x, A_{1}, M_{1}\right) \leqslant C\left|M_{1}\right| ; \\
\left|W^{\infty}\left(x, A_{1}, M_{1}\right)-W^{\infty}\left(x, A_{2}, M_{2}\right)\right| \leqslant C\left|M_{1}-M_{2}\right|
\end{gathered}
$$

and, for every $x_{0} \in \Omega$ and for every $\varepsilon>0$ there exists a $\delta>0$ such that

$$
\left|x-x_{0}\right|<\delta \Rightarrow\left|W^{\infty}\left(x, A_{1}, M_{1}\right)-W^{\infty}\left(x_{0}, A_{1}, M_{1}\right)\right| \leqslant \varepsilon C\left|M_{1}\right| .
$$

Consider now the relaxed energy

$$
I(g, G, \Gamma):=\inf _{\left\{u_{n}\right\} \subset S B V^{2}\left(\Omega ; \mathbb{R}^{d}\right)}\left\{\liminf _{n \rightarrow+\infty} E\left(u_{n}\right): u_{n} \stackrel{L^{1}}{\rightarrow} g, \nabla u_{n} \stackrel{L^{1}}{\rightarrow} G, \nabla^{2} u_{n} \stackrel{*}{\rightarrow} \Gamma\right\} .
$$

The main result of this work reads as follows

Theorem 3.2. For all $(g, G, \Gamma) \in S D^{2}\left(\Omega ; \mathbb{R}^{d}\right)$, under hypotheses (H1) - (H8), we have that

$$
\begin{aligned}
I(g, G, \Gamma)= & \int_{\Omega}\left\{W_{1}(x, G(x)-\nabla g(x))+W_{2}(x, G(x), \nabla G(x), \Gamma(x))\right\} d x \\
& +\int_{S_{g} \cap \Omega} \gamma_{1}\left(x,[g(x)], \nu_{g}(x)\right) d \mathcal{H}^{N-1}(x) \\
& +\int_{S_{G} \cap \Omega} \gamma_{2}\left(x, G(x),[G(x)], \nu_{G}(x)\right) d \mathcal{H}^{N-1}(x),
\end{aligned}
$$

where, for $x \in \Omega, A, \Lambda \in \mathbb{R}^{d \times N}, L, M \in \mathbb{R}^{d \times N \times N}, \lambda \in \mathbb{R}^{d}$ and $\nu \in S^{N-1}$,

$$
\begin{gathered}
W_{1}(x, A)=\inf _{u \in S B V^{2}\left(Q ; \mathbb{R}^{d}\right)}\left\{\int_{S_{u} \cap Q} \Psi_{1}\left(x,[u(y)], \nu_{u}(y)\right) d \mathcal{H}^{N-1}(y):\left.u\right|_{\partial Q}=0, \nabla u=A \text { a.e. in } Q\right\}, \\
\gamma_{1}(x, \lambda, \nu)=\inf _{u \in S B V^{2}\left(Q_{\nu} ; \mathbb{R}^{d}\right)}\left\{\int_{S_{u} \cap Q_{\nu}} \Psi_{1}\left(x,[u(y)], \nu_{u}(y)\right) d \mathcal{H}^{N-1}(y):\left.u\right|_{\partial Q_{\nu}}=\gamma_{(\lambda, \nu)},\right. \\
\left.\nabla u=0 \text { a.e. in } Q_{\nu}\right\},
\end{gathered}
$$

with

$$
\gamma_{(\lambda, \nu)}= \begin{cases}\lambda & \text { if } x \cdot \nu>0 \\ 0 & \text { if } x \cdot \nu<0\end{cases}
$$

and

$$
\begin{gathered}
W_{2}(x, A, L, M)=\inf _{u \in S B V\left(Q ; \mathbb{R}^{d \times N}\right)}\left\{\int_{Q} W(x, A, \nabla u(y)) d y+\int_{S_{u} \cap Q} \Psi_{2}\left(x,[u(y)], \nu_{u}(y)\right) d \mathcal{H}^{N-1}(y):\right. \\
\left.\left.u\right|_{\partial Q}(y)=L \cdot y, \int_{Q} \nabla u(y) d y=M\right\} \\
\gamma_{2}(x, A, \Lambda, \nu)=\inf _{u \in S B V\left(Q_{\nu} ; \mathbb{R}^{d \times N}\right)}\left\{\int_{Q_{\nu}} W^{\infty}(x, A, \nabla u(y)) d y+\int_{S_{u} \cap Q_{\nu}} \Psi_{2}\left(x,[u(y)], \nu_{u}(y)\right) d \mathcal{H}^{N-1}(y):\right. \\
\left.\left.u\right|_{\partial Q_{\nu}}=\gamma_{(\Lambda, \nu)}, \int_{Q_{\nu}} \nabla u(y) d y=0\right\} .
\end{gathered}
$$


SECOND-ORDER STRUCTURED DEFORMATIONS: RELAXATION, INTEGRAL REPRESENTATION AND APPLICATIONS 7

\section{PRELIMINARY RESULTS}

In this section we derive some preliminary results which will be used in the proof of the main theorem.

Lemma 4.1. Let $(g, G, \Gamma) \in S D^{2}\left(\Omega ; \mathbb{R}^{d}\right)$. Then $I(g, G, \Gamma)<+\infty$.

Proof. Let $(g, G, \Gamma) \in S D^{2}\left(\Omega ; \mathbb{R}^{d}\right)$ be given. By applying Theorem 2.4 , there exists $h \in S B V\left(\Omega ; \mathbb{R}^{d \times N}\right)$ such that $\nabla h=\Gamma$ a.e. in $\Omega$ and

$$
\left\|D^{s} h\right\|(\Omega) \leqslant C\|\Gamma\|_{L^{1}\left(\Omega ; \mathbb{R}^{d \times N \times N}\right)}
$$

for some $C=C(N)>0$. By Lemma 2.3 there exists a sequence $\left\{v_{n}\right\} \subset L^{1}\left(\Omega ; \mathbb{R}^{d}\right)$ of piecewise constant functions such that $v_{n} \stackrel{L^{1}}{\rightarrow} G-h$ and

$$
\left\|D v_{n}\right\|(\Omega)=\left\|D^{s} v_{n}\right\|(\Omega) \underset{n \rightarrow+\infty}{\longrightarrow}\|D G-D h\|(\Omega)
$$

Define $w_{n} \in S B V\left(\Omega ; \mathbb{R}^{d \times N}\right)$ by $w_{n}:=v_{n}+h$. We have $w_{n} \rightarrow G$ in $L^{1}\left(\Omega ; \mathbb{R}^{d \times N}\right)$ and $\nabla w_{n}=\Gamma$ a.e. in $\Omega$. By applying again Theorem 2.4 , for every $n \in \mathbb{N}$, there exists $\tilde{h}_{n} \in S B V\left(\Omega ; \mathbb{R}^{d}\right)$ such that $\nabla \tilde{h}_{n}=w_{n}$ a.e. in $\Omega$ and

$$
\left\|D^{s} \tilde{h}_{n}\right\|(\Omega) \leqslant C\left\|w_{n}\right\|_{L^{1}\left(\Omega ; \mathbb{R}^{d \times N}\right)}
$$

By Lemma 2.3, for every $n \in \mathbb{N}$, there exists a sequence $\left\{\bar{h}_{n, m}\right\} \subset L^{1}\left(\Omega ; \mathbb{R}^{d}\right)$ of piecewise constant functions such that $\bar{h}_{n, m} \stackrel{L^{1}}{\rightarrow} g-\tilde{h}_{n}$ as $m \rightarrow+\infty$ and

$$
\left\|D^{s} \bar{h}_{n, m}\right\|(\Omega) \underset{m \rightarrow+\infty}{\longrightarrow}\left\|D g-D \tilde{h}_{n}\right\|(\Omega)
$$

Thus, for every $n \in \mathbb{N}$, there exists $m(n) \in \mathbb{N}$ such that

$$
\left\|\bar{h}_{n, m(n)}-\left(g-\tilde{h}_{n}\right)\right\|_{L^{1}\left(\Omega ; \mathbb{R}^{d}\right)}<\frac{1}{n}, \quad\left|\left\|D^{s} \bar{h}_{n, m(n)}\right\|(\Omega)-\left\|D g-D \tilde{h}_{n}\right\|(\Omega)\right|<\frac{1}{n} .
$$

Hence the sequence $u_{n}:=\tilde{h}_{n}+\bar{h}_{n, m(n)}$ is such that $u_{n} \rightarrow g$ in $L^{1}\left(\Omega ; \mathbb{R}^{d}\right), \nabla u_{n}=w_{n} \rightarrow G$ in $L^{1}\left(\Omega ; \mathbb{R}^{d \times N}\right)$ and $\nabla^{2} u_{n}=\Gamma$, so that it is a competitor for the infimization problem (3.5). 
By the growth assumptions (H1) and (H5), and (4.1), (4.2), (4.3) and (4.4), we can estimate

$$
\begin{aligned}
& I(g, G, \Gamma) \leqslant \liminf _{n \rightarrow+\infty} E\left(u_{n}\right) \\
& \leqslant \liminf _{n \rightarrow+\infty}\left[\int_{\Omega} W\left(x, \nabla u_{n}(x), \nabla^{2} u_{n}(x)\right) d x+\int_{S_{u_{n}}} \Psi_{1}\left(x,\left[u_{n}(x)\right], \nu_{u_{n}}(x)\right) d \mathcal{H}^{N-1}(x)\right. \\
&\left.+\int_{S_{\nabla u_{n}}} \Psi_{2}\left(x,\left[\nabla u_{n}(x)\right], \nu_{\nabla u_{n}}(x)\right) d \mathcal{H}^{N-1}(x)\right] \\
& \leqslant \liminf _{n \rightarrow+\infty}\left[C \int_{\Omega}\left(1+\left|\nabla u_{n}(x)\right|+\left|\nabla^{2} u_{n}(x)\right|\right) d x+K_{1} \int_{S_{u_{n}}}\left|\left[u_{n}(x)\right]\right| d \mathcal{H}^{N-1}(x)\right. \\
&\left.+K_{2} \int_{S_{\nabla u_{n}}}\left|\left[\nabla u_{n}(x)\right]\right| d \mathcal{H}^{N-1}(x)\right] \\
& \leqslant \liminf _{n \rightarrow+\infty}\left[\int_{\Omega} C(1+|G(x)|+|\Gamma(x)|) d x+K_{1}\left\|D^{s} u_{n}\right\|(\Omega)+K_{2}\left\|D^{s}\left(\nabla u_{n}\right)\right\|(\Omega)\right] \\
&+\limsup _{n \rightarrow+\infty} C\left\|w_{n}-G\right\|_{L^{1}\left(\Omega ; \mathbb{R}^{d \times N}\right)} \\
& \leqslant C\left[\mathcal{L}^{N}(\Omega)+\|G\|_{L^{1}\left(\Omega ; \mathbb{R}^{d \times N}\right)}+\|\Gamma\|_{L^{1}\left(\Omega ; \mathbb{R}^{d \times N \times N}\right)}+\|D g\|(\Omega)\right. \\
&\left.+\limsup _{n \rightarrow+\infty}\|D G-D h\|(\Omega)+\limsup _{n \rightarrow+\infty}\left\|w_{n}\right\|_{L^{1}\left(\Omega ; \mathbb{R}^{d \times N}\right)}\right] \\
& \leqslant C\left[\mathcal{L}^{N}(\Omega)+\|G\|_{L^{1}\left(\Omega ; \mathbb{R}^{d \times N}\right)}+\|\Gamma\|_{L^{1}\left(\Omega ; \mathbb{R}^{d \times N \times N}\right)}\right. \\
&\left.+\|D g\|(\Omega)+\|D G\|(\Omega)+\limsup _{n \rightarrow+\infty}\left\|w_{n}-G\right\|_{L^{1}\left(\Omega ; \mathbb{R}^{d \times N}\right)}\right] \\
& \leqslant C\left(1+\|D g\|(\Omega)+\|G\|_{\left.L^{1}\left(\Omega ; \mathbb{R}^{d \times N}\right)+\|D G\|(\Omega)+\|\Gamma\|_{L^{1}\left(\Omega ; \mathbb{R}^{d \times N \times N}\right)}\right) .}\right.
\end{aligned}
$$

Remark 4.2. As the above proof shows, given $(g, G, \Gamma) \in S D^{2}\left(\Omega ; \mathbb{R}^{d}\right)$ there exists a sequence $\left\{u_{n}\right\} \subset S B V^{2}\left(\Omega ; \mathbb{R}^{d}\right)$ such that $u_{n} \rightarrow g$ in $L^{1}\left(\Omega ; \mathbb{R}^{d}\right), \nabla u_{n} \rightarrow G$ in $L^{1}\left(\Omega ; \mathbb{R}^{d \times N}\right)$ and $\nabla^{2} u_{n} \stackrel{*}{\rightarrow} \Gamma$. Our proof is essentially the same as the proof of Theorem 3.2 in [30].

\subsection{Decomposition.}

Theorem 4.3. We may decompose $I(g, G, \Gamma)$ as $I(g, G, \Gamma)=I_{1}(g, G, \Gamma)+I_{2}(G, \Gamma)$, where

$$
\begin{gathered}
I_{1}(g, G, \Gamma):=\inf _{\left\{u_{n}\right\} \subset S B V^{2}\left(\Omega ; \mathbb{R}^{d}\right)}\left\{\liminf _{n \rightarrow+\infty} \int_{S_{u_{n}}} \Psi_{1}\left(x,\left[u_{n}(x)\right], \nu_{u_{n}}(x)\right) d \mathcal{H}^{N-1}(x):\right. \\
\left.u_{n} \stackrel{L^{1}}{\rightarrow} g, \nabla u_{n} \stackrel{L^{1}}{\rightarrow} G, \nabla^{2} u_{n} \stackrel{*}{\rightarrow} \Gamma\right\}
\end{gathered}
$$

and

$$
\begin{aligned}
I_{2}(G, \Gamma):=\quad \inf _{\left\{v_{n}\right\} \subset S B V\left(\Omega ; \mathbb{R}^{d \times N}\right)}\left\{\operatorname { l i m i n f } _ { n \rightarrow + \infty } \left[\int_{\Omega} W\left(x, v_{n}(x), \nabla v_{n}(x)\right) d x\right.\right. \\
\left.\left.\quad+\int_{S_{v_{n}}} \Psi_{2}\left(x,\left[v_{n}(x)\right], \nu_{v_{n}}(x)\right) d \mathcal{H}^{N-1}(x)\right]: v_{n} \stackrel{L^{1}}{\longrightarrow} G, \nabla v_{n} \stackrel{*}{\rightarrow} \Gamma\right\} .
\end{aligned}
$$

Proof. It is clear that

$$
I(g, G, \Gamma) \geqslant I_{1}(g, G, \Gamma)+I_{2}(G, \Gamma)
$$


To show the reverse inequality let $\left\{u_{n}\right\} \subset S B V^{2}\left(\Omega ; \mathbb{R}^{d}\right)$ be such that $u_{n} \stackrel{L^{1}}{\rightarrow} g, \nabla u_{n} \stackrel{L^{1}}{\rightarrow} G$, $\nabla^{2} u_{n} \stackrel{*}{\rightarrow} \Gamma$ and

$$
I_{1}(g, G, \Gamma)=\lim _{n \rightarrow+\infty} \int_{S_{u_{n}}} \Psi_{1}\left(x,\left[u_{n}(x)\right], \nu_{u_{n}}(x)\right) d \mathcal{H}^{N-1}(x)
$$

and let $\left\{v_{n}\right\} \subset S B V^{2}\left(\Omega ; \mathbb{R}^{d \times N}\right)$ be such that $v_{n} \stackrel{L^{1}}{\rightarrow} G, \nabla v_{n} \stackrel{*}{\rightarrow} \Gamma$ and

$$
I_{2}(G, \Gamma)=\lim _{n \rightarrow+\infty}\left[\int_{\Omega} W\left(x, v_{n}(x), \nabla v_{n}(x)\right) d x+\int_{S_{v_{n}}} \Psi_{2}\left(x,\left[v_{n}(x)\right], \nu_{v_{n}}(x)\right) d \mathcal{H}^{N-1}(x)\right] .
$$

By Theorem 2.4 let $\left\{h_{n}\right\} \subset S B V\left(\Omega ; \mathbb{R}^{d}\right)$ be such that $\nabla h_{n}=v_{n}-\nabla u_{n}$ and $\left\|D^{s} h_{n}\right\|(\Omega) \leqslant$ $C\left\|v_{n}-\nabla u_{n}\right\|_{L^{1}\left(\Omega ; \mathbb{R}^{d \times N)}\right.}$, and by Lemma 2.3 let $\tilde{h}_{n}$ be a sequence of piecewise constant functions with $\left\|\tilde{h}_{n}-h_{n}\right\|_{L^{1}}<\frac{1}{n}$ and $\left|\left\|D \tilde{h}_{n}\right\|(\Omega)-\left\|D h_{n}\right\|(\Omega)\right|<\frac{1}{n}$. Define $\left\{w_{n}\right\} \subset S B V^{2}\left(\Omega ; \mathbb{R}^{d}\right)$ by

$$
w_{n}:=u_{n}+h_{n}-\tilde{h}_{n} .
$$

Then $w_{n} \stackrel{L^{1}}{\rightarrow} g, \nabla w_{n}=v_{n} \stackrel{L^{1}}{\longrightarrow} G, \nabla^{2} w_{n}=\nabla v_{n} \stackrel{*}{\rightarrow} \Gamma$ and so, by (H8) and (H5),

$$
\begin{aligned}
I(g, G, \Gamma) \leqslant & \liminf _{n \rightarrow+\infty}\left[\int_{\Omega} W\left(x, \nabla w_{n}(x), \nabla^{2} w_{n}(x)\right) d x+\int_{S_{w_{n}}} \Psi_{1}\left(x,\left[w_{n}(x)\right], \nu_{w_{n}}(x)\right) d \mathcal{H}^{N-1}(x)\right. \\
& \left.\quad+\int_{S_{\nabla w_{n}}} \Psi_{2}\left(x,\left[\nabla w_{n}(x)\right], \nu_{\nabla w_{n}}(x)\right) d \mathcal{H}^{N-1}(x)\right] \\
& \leqslant \lim _{n \rightarrow+\infty}\left[\int_{\Omega} W\left(x, \nabla v_{n}(x), \nabla v_{n}(x)\right) d x+\int_{S_{v_{n}}} \Psi_{2}\left(x,\left[v_{n}(x)\right], \nu_{v_{n}}(x)\right) d \mathcal{H}^{N-1}(x)\right] \\
& +\lim _{n \rightarrow+\infty} \int_{S_{u_{n}}} \Psi_{1}\left(x,\left[u_{n}(x)\right], \nu_{u_{n}}(x)\right) d \mathcal{H}^{N-1}(x) \\
& +\limsup _{n \rightarrow+\infty} \int_{S_{h_{n}} \cup S_{\tilde{h}_{n}}} \Psi_{1}\left(x,\left[h_{n}-\tilde{h}_{n}\right](x), \nu_{h_{n}-\tilde{h}_{n}}(x)\right) d \mathcal{H}^{N-1}(x) \\
& \leqslant I_{2}(G, \Gamma)+I_{1}(g, G, \Gamma)+\limsup _{n \rightarrow+\infty} C \int_{S_{h_{n}} \cup S_{\tilde{h}_{n}}}\left|\left[h_{n}-\tilde{h}_{n}\right](x)\right| d \mathcal{H}^{N-1}(x) \\
& \leqslant I_{2}(G, \Gamma)+I_{1}(g, G, \Gamma)+\limsup _{n \rightarrow+\infty} C \int_{\Omega}\left|v_{n}(x)-\nabla u_{n}(x)\right| d x \\
& =I_{2}(G, \Gamma)+I_{1}(g, G, \Gamma),
\end{aligned}
$$

where we have used the properties of the functions $u_{n}, v_{n}, h_{n}$ and $\tilde{h}_{n}$.

4.2. Localization. In this section we localize the functionals $I_{1}$ and $I_{2}$ and show that they are Radon measures. For each $U \in \mathcal{O}(\Omega)$ we define the localized functionals

$$
\begin{gathered}
I_{1}(g, G, \Gamma, U):=\inf _{\left\{u_{n}\right\} \subset S B V^{2}\left(U ; \mathbb{R}^{d}\right)}\left\{\liminf _{n \rightarrow+\infty} \int_{S_{u_{n}} \cap U} \Psi_{1}\left(x,\left[u_{n}(x)\right], \nu_{u_{n}}(x)\right) d \mathcal{H}^{N-1}(x):\right. \\
\left.u_{n} \stackrel{L^{1}}{\rightarrow} g, \nabla u_{n} \stackrel{L^{1}}{\rightarrow} G, \nabla^{2} u_{n} \stackrel{*}{\rightarrow} \Gamma\right\}
\end{gathered}
$$

and

$$
\begin{aligned}
I_{2}(G, \Gamma, U) & :=\inf _{\left\{v_{n}\right\} \subset S B V\left(U ; \mathbb{R}^{d \times N}\right)}\left\{\operatorname { l i m i n f } _ { n \rightarrow + \infty } \left[\int_{U} W\left(x, v_{n}(x), \nabla v_{n}(x)\right) d x\right.\right. \\
& \left.\left.+\int_{S_{v_{n}} \cap U} \Psi_{2}\left(x,\left[v_{n}(x)\right], \nu_{v_{n}}(x)\right) d \mathcal{H}^{N-1}(x)\right]: v_{n} \stackrel{L^{1}}{\rightarrow} G, \nabla v_{n} \stackrel{*}{\rightarrow} \Gamma\right\} .
\end{aligned}
$$


It is clear that localized versions of the upper bound (4.5) still hold, namely

$$
\begin{gathered}
I_{1}(g, G, \Gamma, U) \leqslant C\left[\|G\|_{L^{1}\left(U ; \mathbb{R}^{d \times N}\right)}+\|D g\|(U)\right], \\
I_{2}(G, \Gamma, U) \leqslant C\left[1+\|G\|_{L^{1}\left(U ; \mathbb{R}^{d \times N}\right)}+\|\Gamma\|_{L^{1}\left(U ; \mathbb{R}^{d \times N \times N}\right)}+\|D G\|(U)\right] .
\end{gathered}
$$

We will now prove that $I_{1}(g, G, \Gamma, \cdot)\left\lfloor\mathcal{O}(\Omega)\right.$ and $I_{2}(G, \Gamma, \cdot)\lfloor\mathcal{O}(\Omega)$ are Radon measures. For this purpose we first show that these functionals are nested subadditive.

Lemma 4.4. Let $U, V, W \in \mathcal{O}(\Omega)$ be such that $U \subset \subset V \subset W$. Then

$$
\begin{gathered}
I_{1}(g, G, \Gamma, W) \leqslant I_{1}(g, G, \Gamma, V)+I_{1}(g, G, \Gamma, W \backslash \bar{U}), \\
I_{2}(G, \Gamma, W) \leqslant I_{2}(G, \Gamma, V)+I_{2}(G, \Gamma, W \backslash \bar{U}) .
\end{gathered}
$$

Proof. We provide the details of the proof only for $I_{1}$ since for $I_{2}$ it is analogous.

Let $u_{n} \in S B V^{2}\left(V ; \mathbb{R}^{d}\right)$ and $v_{n} \in S B V^{2}\left(W \backslash \bar{U} ; \mathbb{R}^{d}\right)$ be two sequences such that $u_{n} \rightarrow g$ in $L^{1}\left(V ; \mathbb{R}^{d}\right), \nabla u_{n} \rightarrow G$ in $L^{1}\left(V ; \mathbb{R}^{d \times N}\right), \nabla^{2} u_{n} \stackrel{*}{\rightarrow} \Gamma$ in $\mathcal{M}\left(V ; \mathbb{R}^{d \times N \times N}\right), v_{n} \rightarrow g$ in $L^{1}\left(W \backslash \bar{U} ; \mathbb{R}^{d}\right)$, $\nabla v_{n} \rightarrow G$ in $L^{1}\left(W \backslash \bar{U} ; \mathbb{R}^{d \times N}\right), \nabla^{2} v_{n} \stackrel{*}{\rightarrow} \Gamma$ in $\mathcal{M}\left(W \backslash \bar{U} ; \mathbb{R}^{d \times N \times N}\right)$, and that, in addition,

$$
I_{1}(g, G, \Gamma, V)=\lim _{n \rightarrow+\infty} \int_{S_{u_{n}} \cap V} \Psi_{1}\left(x,\left[u_{n}(x)\right], \nu_{u_{n}}(x)\right) d \mathcal{H}^{N-1}(x)
$$

and

$$
I_{1}(g, G, \Gamma, W \backslash \bar{U})=\lim _{n \rightarrow+\infty} \int_{S_{v_{n}} \cap(W \backslash \bar{U})} \Psi_{1}\left(x,\left[v_{n}(x)\right], \nu_{v_{n}}(x)\right) d \mathcal{H}^{N-1}(x) .
$$

Note that

$$
u_{n}-v_{n} \rightarrow 0 \text { in } L^{1}\left(V \cap(W \backslash \bar{U}) ; \mathbb{R}^{d}\right)
$$

and

$$
\begin{gathered}
\nabla u_{n}-\nabla v_{n} \rightarrow 0 \text { in } L^{1}\left(V \cap(W \backslash \bar{U}) ; \mathbb{R}^{d \times N}\right), \\
\nabla^{2} u_{n}-\nabla^{2} v_{n} \stackrel{*}{\rightarrow} 0 \text { in } \mathcal{M}\left(V \cap(W \backslash \bar{U}) ; \mathbb{R}^{d \times N \times N}\right) .
\end{gathered}
$$

For $\delta>0$ define

$$
U_{\delta}:=\{x \in V: \operatorname{dist}(x, U)<\delta\} .
$$

For $x \in W$ let $d(x):=\operatorname{dist}(x, U)$. Since the distance function to a fixed set is Lipschitz continuous (see [33, Exercise 1.1]), we can apply the change of variables formula [21, Section 3.4.3, Theorem 2], to obtain

$$
\int_{U_{\delta} \backslash \bar{U}}\left|u_{n}(x)-v_{n}(x)\right||\operatorname{det} \nabla d(x)| d x=\int_{0}^{\delta}\left[\int_{d^{-1}(y)}\left|u_{n}(x)-v_{n}(x)\right| d \mathcal{H}^{N-1}(x)\right] d y
$$

and, as $|\operatorname{det} \nabla d|$ is bounded and (4.12) holds, it follows that for almost every $\rho \in[0, \delta]$ we have

$$
\lim _{n \rightarrow+\infty} \int_{d^{-1}(\rho)}\left|u_{n}(x)-v_{n}(x)\right| d \mathcal{H}^{N-1}(x)=\lim _{n \rightarrow+\infty} \int_{\partial U_{\rho}}\left|u_{n}(x)-v_{n}(x)\right| d \mathcal{H}^{N-1}(x)=0 .
$$

Fix $\rho_{0} \in[0, \delta]$ such that $\left\|\Gamma \chi_{V}\right\|\left(\partial U_{\rho_{0}}\right)=0,\left\|\Gamma \chi_{W \backslash \bar{U}}\right\|\left(\partial U_{\rho_{0}}\right)=0$ and such that (4.13) holds. We observe that $U_{\rho_{0}}$ is a set with locally Lipschitz boundary since it is a level set of a Lipschitz function (see, e.g., [21]). Hence we can consider $u_{n}, v_{n}, \nabla u_{n}, \nabla v_{n}$ on $\partial U_{\rho_{0}}$ in the sense of traces and define

$$
w_{n}= \begin{cases}u_{n} & \text { in } \bar{U}_{\rho_{0}} \\ v_{n} & \text { in } W \backslash \bar{U}_{\rho_{0}} .\end{cases}
$$


SECOND-ORDER STRUCTURED DEFORMATIONS: RELAXATION, INTEGRAL REPRESENTATION AND APPLICATIONS1

Then, by the choice of $\rho_{0}, w_{n}$ is admissible for $I_{1}(g, G, \Gamma, W)$ so, by (H5), (4.12) and (4.13), we obtain

$$
\begin{aligned}
& I_{1}(g, G, \Gamma, W) \leqslant \liminf _{n \rightarrow+\infty} \int_{S_{w_{n}} \cap W} \Psi_{1}\left(x,\left[w_{n}(x)\right], \nu_{w_{n}}(x)\right) d \mathcal{H}^{N-1}(x) \\
& \leqslant \liminf _{n \rightarrow+\infty} {\left[\int_{S_{u_{n}} \cap V} \Psi_{1}\left(x,\left[u_{n}(x)\right], \nu_{u_{n}}(x)\right) d \mathcal{H}^{N-1}(x)\right.} \\
&+\int_{S_{v_{n}} \cap(W \backslash \bar{U})} \Psi_{1}\left(x,\left[v_{n}(x)\right], \nu_{v_{n}}(x)\right) d \mathcal{H}^{N-1}(x) \\
&\left.+\int_{S_{w_{n}} \cap \partial U_{\rho_{0}}} C\left|u_{n}(x)-v_{n}(x)\right| d \mathcal{H}^{N-1}(x)\right] \\
&=I_{1}(g, G, \Gamma, V)+I_{1}(g, G, \Gamma, W \backslash \bar{U}),
\end{aligned}
$$

which concludes the proof.

Theorem 4.5. Assume that hypotheses (H1) and (H5) hold. Then $I_{1}(g, G, \Gamma, \cdot)\lfloor\mathcal{O}(\Omega)$ and $I_{2}(G, \Gamma, \cdot)\left\lfloor\mathcal{O}(\Omega)\right.$ are Radon measures, absolutely continuous with respect to $\mathcal{L}^{N}+\mathcal{H}^{N-1}\left\lfloor S_{g}\right.$ and to $\mathcal{L}^{N}+\mathcal{H}^{N-1}\left\lfloor S_{G}\right.$, respectively.

Proof. Let $u_{n} \in S B V^{2}\left(\Omega ; \mathbb{R}^{d}\right)$ be such that $u_{n} \rightarrow g$ in $L^{1}\left(\Omega ; \mathbb{R}^{d}\right), \nabla u_{n} \rightarrow G$ in $L^{1}\left(\Omega ; \mathbb{R}^{d \times N}\right)$, $\nabla^{2} u_{n} \stackrel{*}{\rightarrow} \Gamma$ in $\mathcal{M}\left(\Omega ; \mathbb{R}^{d \times N \times N}\right)$ and

$$
I_{1}(g, G, \Gamma, \Omega)=\lim _{n \rightarrow+\infty} \int_{S_{u_{n}} \cap \Omega} \Psi_{1}\left(x,\left[u_{n}(x)\right], \nu_{u_{n}}(x)\right) d \mathcal{H}^{N-1}(x) .
$$

For every Borel set $B \subset \bar{\Omega}$ define the sequence of measures

$$
\mu_{n}(B):=\int_{S_{u_{n} \cap B}} \Psi_{1}\left(x,\left[u_{n}(x)\right], \nu_{u_{n}}(x)\right) d \mathcal{H}^{N-1}(x) .
$$

By (H5) this sequence of non-negative Radon measures is uniformly bounded in $\mathcal{M}(\bar{\Omega})$ and thus, upon passing if necessary to a subsequence, we conclude that

$$
\mu_{n} \stackrel{*}{\rightarrow} \mu \text { in } \mathcal{M}(\bar{\Omega}) \text {. }
$$

In particular,

$$
\mu(\bar{\Omega})=I_{1}(g, G, \Gamma, \Omega)
$$

We want to show that, for all $V \in \mathcal{O}(\Omega)$,

$$
\mu(V)=I_{1}(g, G, \Gamma, V) .
$$

Let $V \in \mathcal{O}(\Omega)$, let $\varepsilon>0$ and choose $W \subset \subset V$ such that $\mu(V \backslash W)<\varepsilon$. Since $W \subset \subset V \subset \bar{\Omega}$, by the nested subadditivity property it follows that

$$
\begin{aligned}
\mu(\bar{\Omega}) & =I_{1}(g, G, \Gamma, \Omega) \\
& \leqslant I_{1}(g, G, \Gamma, V)+I_{1}(g, G, \Gamma, \Omega \backslash \bar{W}) \\
& \leqslant I_{1}(g, G, \Gamma, V)+\mu(\overline{\Omega \backslash \bar{W}}),
\end{aligned}
$$

and so,

$$
\begin{aligned}
\mu(V) & \leqslant \mu(W)+\varepsilon \\
& =\mu(\bar{\Omega})-\mu(\bar{\Omega} \backslash W)+\varepsilon \\
& \leqslant I_{1}(g, G, \Gamma, \Omega)-I_{1}(g, G, \Gamma, \Omega \backslash \bar{W})+\varepsilon \\
& \leqslant I_{1}(g, G, \Gamma, V)+\varepsilon .
\end{aligned}
$$

Thus, letting $\varepsilon \rightarrow 0^{+}$, we conclude that

$$
\mu(V) \leqslant I_{1}(g, G, \Gamma, V) .
$$


To prove the reverse inequality define, for $U \in \mathcal{O}(\Omega)$,

$$
\lambda(U):=\int_{U}(|\nabla g(x)|+|G(x)|) d x+\left\|D^{s} g\right\|(U) .
$$

Let $K \subset \subset V$ be a compact set such that $\lambda(V \backslash K)<\varepsilon$ and choose an open set $W$ such that $K \subset \subset W \subset \subset V$. Lemma 4.4, (4.16) and (4.8) yield

$$
\begin{aligned}
I_{1}(g, G, \Gamma, V) & \leqslant I_{1}(g, G, \Gamma, W)+I_{1}(g, G, \Gamma, V \backslash K) \\
& \leqslant \liminf _{n \rightarrow+\infty} \mu_{n}(W)+C \lambda(V \backslash K) \\
& \leqslant \limsup _{n \rightarrow+\infty} \mu_{n}(\bar{W})+C \varepsilon \\
& \leqslant \mu(\bar{W})+C \varepsilon \\
& \leqslant \mu(V)+C \varepsilon,
\end{aligned}
$$

so to conclude the result it suffices to let $\varepsilon \rightarrow 0^{+}$.

In the case of $I_{2}$ the proof is analogous, using hypotheses (H1) and (H5), (4.9) and the nested subadditivity property (4.11).

We now define

$$
\begin{aligned}
\tilde{I}_{2}(G, \Gamma):= & \inf _{v_{n} \subset S B V\left(\Omega ; \mathbb{R}^{d \times N}\right)}\left\{\operatorname { l i m i n f } _ { n \rightarrow + \infty } \left[\int_{\Omega} W\left(x, G(x), \nabla v_{n}(x)\right) d x\right.\right. \\
& \left.\left.+\int_{S_{v_{n}} \cap \Omega} \Psi_{2}\left(x,\left[v_{n}(x)\right], \nu_{v_{n}}(x)\right) d \mathcal{H}^{N-1}(x)\right]: v_{n} \stackrel{L^{1}}{\longrightarrow} G, \nabla v_{n} \stackrel{*}{\rightarrow} \Gamma\right\} .
\end{aligned}
$$

Proposition 4.6. Let $(G, \Gamma) \in B V\left(\Omega ; \mathbb{R}^{d \times N}\right) \times L^{1}\left(\Omega ; \mathbb{R}^{d \times N \times N}\right)$. Then we have that

$$
I_{2}(G, \Gamma)=\tilde{I}_{2}(G, \Gamma) \text {. }
$$

Proof. Let $\left\{v_{n}\right\} \subset S B V\left(\Omega ; \mathbb{R}^{d \times N}\right)$ be such that $v_{n} \rightarrow G$ in $L^{1}\left(\Omega ; \mathbb{R}^{d \times N}\right), \nabla v_{n} \stackrel{*}{\rightarrow} \Gamma$ and

$$
I_{2}(G, \Gamma)=\lim _{n \rightarrow+\infty}\left[\int_{\Omega} W\left(x, v_{n}(x), \nabla v_{n}(x)\right) d x+\int_{S_{v_{n}} \cap \Omega} \Psi_{2}\left(x,\left[v_{n}(x)\right], \nu_{v_{n}}(x)\right) d \mathcal{H}^{N-1}(x)\right] .
$$

By (H2) it follows that

$$
\begin{aligned}
\tilde{I}_{2}(G, \Gamma) & \leqslant \lim _{n \rightarrow+\infty}\left[\int_{\Omega} W\left(x, G(x), \nabla v_{n}(x)\right) d x+\int_{S_{v_{n}} \cap \Omega} \Psi_{2}\left(x,\left[v_{n}(x)\right], \nu_{v_{n}}(x)\right) d \mathcal{H}^{N-1}(x)\right] \\
& \leqslant \limsup _{n \rightarrow+\infty}\left[\int_{\Omega} W\left(x, G(x), \nabla v_{n}(x)\right)-W\left(x, v_{n}(x), \nabla v_{n}(x)\right) d x\right] \\
& +\lim _{n \rightarrow+\infty}\left[\int_{\Omega} W\left(x, v_{n}(x), \nabla v_{n}(x)\right) d x+\int_{S_{v_{n}} \cap \Omega} \Psi_{2}\left(x,\left[v_{n}(x)\right], \nu_{v_{n}}(x)\right) d \mathcal{H}^{N-1}(x)\right] \\
& \leqslant \limsup _{n \rightarrow+\infty} C \int_{\Omega}\left|G(x)-v_{n}(x)\right| d x+I_{2}(G, \Gamma)=I_{2}(G, \Gamma) .
\end{aligned}
$$

The reverse inequality is proved similarly.

A standard diagonalization argument yields the following lower semicontinuity property of both $I_{1}$ and $I_{2}$.

Proposition 4.7. Let $(g, G, \Gamma) \in S D^{2}\left(\Omega ; \mathbb{R}^{d}\right)$ and $g_{n} \in S B V^{2}\left(\Omega ; \mathbb{R}^{d}\right), G_{n} \in S B V\left(\Omega ; \mathbb{R}^{d \times N}\right)$ be such that $g_{n} \rightarrow g$ in $L^{1}\left(\Omega ; \mathbb{R}^{d}\right)$ and $G_{n} \rightarrow G$ in $L^{1}\left(\Omega ; \mathbb{R}^{d \times N}\right)$. Then

$$
I_{1}(g, G, \Gamma, \Omega) \leqslant \liminf _{n \rightarrow+\infty} I_{1}\left(g_{n}, G, \Gamma, \Omega\right)
$$

and

$$
I_{2}(G, \Gamma, \Omega) \leqslant \liminf _{n \rightarrow+\infty} I_{2}\left(G_{n}, \Gamma, \Omega\right)
$$


4.3. Properties of the density functions. In order to prove the upper bound inequality for the surface energy terms of both $I_{1}$ and $I_{2}$ we need the following properties of the density functions $W_{1}, W_{2}, \gamma_{1}$ and $\gamma_{2}$.

Proposition 4.8. $\quad$ i) $W_{1}(x, 0)=0, \forall x \in \Omega$;

ii) $\left|W_{1}(x, A)-W_{1}(x, B)\right| \leqslant C|A-B|, \forall x \in \Omega, \forall A, B \in \mathbb{R}^{d \times N}$.

Proof. The proof of $i$ ) is immediate by noticing that the function $u=0$ is admissible for $W_{1}(x, 0)$. To prove $\left.i i\right)$ we will show that $W_{1}(x, B) \leqslant W_{1}(x, A)+C|A-B|, \forall x \in \Omega, \forall A, B \in \mathbb{R}^{d \times N}$; the reverse inequality follows by interchanging the roles of $A$ and $B$.

Fix $\varepsilon>0$ and let $u \in S B V^{2}\left(Q ; \mathbb{R}^{d}\right)$ be such that $\left.u\right|_{\partial Q}=0, \nabla u=A$ a.e. in $Q$ and

$$
\int_{S_{u} \cap Q} \Psi_{1}\left(x,[u(y)], \nu_{u}(y)\right) d \mathcal{H}^{N-1}(y) \leqslant W_{1}(x, A)+\varepsilon .
$$

By Lemma 2.5, let $v \in S B V^{2}\left(Q ; \mathbb{R}^{d}\right)$ be such that $\left.v\right|_{\partial Q}=0, \nabla v=B-A$ a.e. in $Q$ and $\left|D^{s} v\right|(Q) \leqslant C|B-A|$, and define $w=u+v$. Then $w$ is admissible for $W_{1}(x, B)$ so by (H8) and (H5),

$$
\begin{aligned}
W_{1}(x, B) & \leqslant \int_{S_{w} \cap Q} \Psi_{1}\left(x,[w(y)], \nu_{w}(y)\right) d \mathcal{H}^{N-1}(y) \\
& \leqslant \int_{S_{u} \cap Q} \Psi_{1}\left(x,[u(y)], \nu_{u}(y)\right) d \mathcal{H}^{N-1}(y)+\int_{S_{v} \cap Q} \Psi_{1}\left(x,[v(y)], \nu_{v}(y)\right) d \mathcal{H}^{N-1}(y) \\
& \leqslant W_{1}(x, A)+\varepsilon+C\left|D^{s} v\right|(Q) \leqslant W_{1}(x, A)+\varepsilon+C|B-A| .
\end{aligned}
$$

Hence the result follows by letting $\varepsilon \rightarrow 0^{+}$.

Proposition 4.9. i) $\gamma_{1}(x, \lambda, \nu) \leqslant C|\lambda|, \forall(x, \lambda, \nu) \in \Omega \times \mathbb{R}^{d} \times S^{N-1}$;

ii) for every $x_{0} \in \Omega$ and for every $\varepsilon>0$ there exists $\delta>0$ such that $\left|x-x_{0}\right|<\delta \Rightarrow\left|\gamma_{1}\left(x_{0}, \lambda, \nu\right)-\gamma_{1}(x, \lambda, \nu)\right| \leqslant \varepsilon C(1+|\lambda|), \forall(x, \lambda, \nu) \in \Omega \times \mathbb{R}^{d} \times S^{N-1} ;$

iii) $\left|\gamma_{1}(x, \lambda, \nu)-\gamma_{1}\left(x, \lambda^{\prime}, \nu\right)\right| \leqslant C\left|\lambda-\lambda^{\prime}\right|, \forall(x, \lambda, \nu),\left(x, \lambda^{\prime}, \nu\right) \in \Omega \times \mathbb{R}^{d} \times S^{N-1}$;

iv) $\gamma_{1}$ is upper semicontinuous in $\Omega \times \mathbb{R}^{d} \times S^{N-1}$.

Proof. The proof of $i$ ) follows immediately from the fact that the function $\gamma_{(\lambda, \nu)}$ is admissible for $\gamma_{1}(x, \lambda, \nu)$ and from hypotheses (H5).

To prove $i i$ ) fix $x_{0} \in \Omega$ and $\varepsilon>0$. By (H6) let $\delta>0$ be such that

$$
\left|x-x_{0}\right|<\delta \Rightarrow\left|\Psi_{1}\left(x_{0}, \lambda, \nu\right)-\Psi_{1}(x, \lambda, \nu)\right| \leqslant \varepsilon C|\lambda| .
$$

Let $u_{n} \in S B V^{2}\left(Q_{\nu} ; \mathbb{R}^{d}\right)$ be such that $\left.u_{n}\right|_{\partial Q_{\nu}}=\gamma_{(\lambda, \nu)}, \nabla u_{n}=0$ a.e. in $Q_{\nu}$ and

$$
\int_{S_{u_{n}} \cap Q_{\nu}} \Psi_{1}\left(x_{0},\left[u_{n}(y)\right], \nu_{u_{n}}(y)\right) d \mathcal{H}^{N-1}(y) \leqslant \gamma_{1}\left(x_{0}, \lambda, \nu\right)+\frac{1}{n} .
$$

By (H5) and $i$ ) we have

$$
\begin{aligned}
\int_{S_{u_{n}} \cap Q_{\nu}}\left|\left[u_{n}(y)\right]\right| d \mathcal{H}^{N-1}(y) & \leqslant C \int_{S_{u_{n}} \cap Q_{\nu}} \Psi_{1}\left(x_{0},\left[u_{n}(y)\right], \nu_{u_{n}}(y)\right) d \mathcal{H}^{N-1}(y) \\
& \leqslant C\left(\gamma_{1}\left(x_{0}, \lambda, \nu\right)+\frac{1}{n}\right) \leqslant C(1+|\lambda|) .
\end{aligned}
$$

Hence, if $\left|x-x_{0}\right|<\delta$, it follows by (4.17) and (4.18) that

$$
\begin{aligned}
& \gamma_{1}(x, \lambda, \nu)-\gamma_{1}\left(x_{0}, \lambda, \nu\right) \\
& \leqslant \int_{S_{u_{n}} \cap Q_{\nu}} \Psi_{1}\left(x,\left[u_{n}(y)\right], \nu_{u_{n}}(y)\right) d \mathcal{H}^{N-1}(y)-\int_{S_{u_{n}} \cap Q_{\nu}} \Psi_{1}\left(x_{0},\left[u_{n}(y)\right], \nu_{u_{n}}(y)\right) d \mathcal{H}^{N-1}(y)+\frac{1}{n} \\
& \leqslant \int_{S_{u_{n}} \cap Q_{\nu}} \varepsilon C\left|\left[u_{n}(y)\right]\right| d \mathcal{H}^{N-1}(y)+\frac{1}{n} \\
& \leqslant \varepsilon C(1+|\lambda|)+\frac{1}{n} .
\end{aligned}
$$


Letting $n \rightarrow+\infty$ we conclude that

$$
\gamma_{1}(x, \lambda, \nu)-\gamma_{1}\left(x_{0}, \lambda, \nu\right) \leqslant \varepsilon C(1+|\lambda|) .
$$

Changing the roles of $x$ and $x_{0}$ we obtain the result.

We now prove iii). Let $u \in S B V^{2}\left(Q_{\nu} ; \mathbb{R}^{d}\right)$ be such that $\left.u\right|_{\partial Q_{\nu}}=\gamma_{(\lambda, \nu)}, \nabla u=0$ a.e. in $Q_{\nu}$ and

$$
\int_{S_{u} \cap Q_{\nu}} \Psi_{1}\left(x,[u(y)], \nu_{u}(y)\right) d \mathcal{H}^{N-1}(y) \leqslant \gamma_{1}(x, \lambda, \nu)+\varepsilon .
$$

Let $v=\gamma_{\left(\lambda^{\prime}, \nu\right)}-\gamma_{(\lambda, \nu)}$ and define $w=u+v$. Since $w$ is admissible for $\gamma_{1}\left(x, \lambda^{\prime}, \nu\right)$ we have by (H8) and (H5),

$$
\begin{aligned}
\gamma_{1}\left(x, \lambda^{\prime}, \nu\right) & \leqslant \int_{S_{w} \cap Q_{\nu}} \Psi_{1}\left(x,[w(y)], \nu_{w}(y)\right) d \mathcal{H}^{N-1}(y) \\
& \leqslant \int_{S_{u} \cap Q_{\nu}} \Psi_{1}\left(x,[u(y)], \nu_{u}(y)\right) d \mathcal{H}^{N-1}(y)+\int_{S_{v} \cap Q_{\nu}} \Psi_{1}\left(x,[v(y)], \nu_{v}(y)\right) d \mathcal{H}^{N-1}(y) \\
& \leqslant \gamma_{1}(x, \lambda, \nu)+\varepsilon+\int_{\left\{y \in Q_{\nu}: y \cdot \nu=0\right\}} \Psi_{1}\left(x, \lambda^{\prime}-\lambda, \nu\right) d \mathcal{H}^{N-1}(y) \\
& \leqslant \gamma_{1}(x, \lambda, \nu)+\varepsilon+C\left|\lambda^{\prime}-\lambda\right|,
\end{aligned}
$$

so to prove the first inequality it suffices to let $\varepsilon \rightarrow 0^{+}$. The other inequality is obtained in a similar fashion.

To prove $i v)$, taking into account the result of $i i i)$ it suffices to show that $(x, \nu) \rightarrow \gamma_{1}(x, \lambda, \nu)$ is upper semicontinuous, for every $\lambda \in \mathbb{R}^{d}$. By a change of variables argument, choosing a rotation $R$ such that $R e_{N}=\nu$, it is easy to see that

$$
\gamma_{1}(x, \lambda, \nu)=\inf _{u \in S B V^{2}\left(Q ; \mathbb{R}^{d}\right)}\left\{\int_{S_{u} \cap Q} \Psi_{1}\left(x,[u(y)], \nu_{u}(y)\right) d \mathcal{H}^{N-1}(y):\left.u\right|_{\partial Q}=\gamma_{\left(\lambda, e_{N}\right)}, \nabla u=0 \text { a.e. in } Q\right\} .
$$

Let $\left(x_{n}, \nu_{n}\right) \rightarrow(x, \nu)$. Given $\varepsilon>0$, let $u_{\varepsilon} \in S B V^{2}\left(Q ; \mathbb{R}^{d}\right)$ be such that $\left.u_{\varepsilon}\right|_{\partial Q}=\gamma_{\left(\lambda, e_{N}\right)}, \nabla u_{\varepsilon}=0$ a.e. in $Q$ and

$$
\left|\gamma_{1}(x, \lambda, \nu)-\int_{S_{u_{\varepsilon}} \cap Q} \Psi_{1}\left(x,\left[u_{\varepsilon}(y)\right], \nu_{u_{\varepsilon}}(y)\right) d \mathcal{H}^{N-1}(y)\right|<\varepsilon .
$$

Let $K$ be a compact subset of $\Omega$ containing a neighborhood of $x$ and choose $\delta>0$ such that (H6) is satisfied uniformly in $K$, i.e.

$$
y, y^{\prime} \in K,\left|y-y^{\prime}\right|<\delta \Rightarrow\left|\Psi_{1}(y, \lambda, \nu)-\Psi_{1}\left(y^{\prime}, \lambda, \nu\right)\right| \leqslant \varepsilon C|\lambda|,
$$

for all $(\lambda, \nu) \in \mathbb{R}^{d} \times S^{N-1}$. Choosing rotations $R_{n}$ such that $R_{n} e_{N}=\nu_{n}, R_{n} \rightarrow R$, by (4.21), (H5) and (4.20) we have that

$$
\begin{aligned}
& \left|\int_{S_{u_{\varepsilon}} \cap Q} \Psi_{1}\left(x,\left[u_{\varepsilon}(y)\right], \nu_{u_{\varepsilon}}(y)\right) d \mathcal{H}^{N-1}(y)-\int_{S_{u_{\varepsilon}} \cap Q} \Psi_{1}\left(x_{n},\left[u_{\varepsilon}(y)\right], \nu_{u_{\varepsilon}}(y)\right) d \mathcal{H}^{N-1}(y)\right| \\
& \leqslant \int_{S_{u_{\varepsilon}} \cap Q} \varepsilon C\left|\left[u_{\varepsilon}(y)\right]\right| d \mathcal{H}^{N-1}(y) \\
& \leqslant \varepsilon C \int_{S_{u_{\varepsilon} \cap Q}} \Psi_{1}\left(x,\left[u_{\varepsilon}(y)\right], \nu_{u_{\varepsilon}}(y)\right) d \mathcal{H}^{N-1}(y) \\
& \leqslant \varepsilon C\left(\varepsilon+\gamma_{1}(x, \lambda, \nu)\right)=O(\varepsilon) .
\end{aligned}
$$

Thus, by (4.19) and (4.20),

$$
\begin{aligned}
\gamma_{1}\left(x_{n}, \lambda, \nu_{n}\right) & \leqslant \int_{S_{u_{\varepsilon}} \cap Q} \Psi_{1}\left(x_{n},\left[u_{\varepsilon}(y)\right], \nu_{u_{\varepsilon}}(y)\right) d \mathcal{H}^{N-1}(y) \\
& \leqslant O(\varepsilon)+\int_{S_{u_{\varepsilon}} \cap Q} \Psi_{1}\left(x,\left[u_{\varepsilon}(y)\right], \nu_{u_{\varepsilon}}(y)\right) d \mathcal{H}^{N-1}(y) \\
& \leqslant O(\varepsilon)+\gamma_{1}(x, \lambda, \nu) .
\end{aligned}
$$


SECOND-ORDER STRUCTURED DEFORMATIONS: RELAXATION, INTEGRAL REPRESENTATION AND APPLICATIONS5

Therefore, letting $\varepsilon \rightarrow 0^{+}$, we conclude that

$$
\limsup _{n \rightarrow+\infty} \gamma_{1}\left(x_{n}, \lambda, \nu_{n}\right) \leqslant \gamma_{1}(x, \lambda, \nu) .
$$

Remark 4.10. $\gamma_{1}(x, \lambda, \nu)$ can be extended to $\Omega \times \mathbb{R}^{d} \times \mathbb{R}^{N}$ as a positively homogeneous of degree one function in the third variable in the following way

$$
\gamma_{1}(x, \lambda, \theta)= \begin{cases}|\theta| \gamma_{1}\left(x, \lambda, \frac{\theta}{|\theta|}\right), & \text { if } \theta \in \mathbb{R}^{N} \backslash\{0\} \\ 0, & \text { if } \theta=0 .\end{cases}
$$

By Proposition 4.9 this extension is upper semicontinuous in $\Omega \times \mathbb{R}^{d} \times \mathbb{R}^{N}$ and satisfies

$$
\gamma_{1}(x, \lambda, \theta) \leqslant C|\lambda||\theta|, \forall(x, \lambda, \theta) \in \Omega \times \mathbb{R}^{d} \times \mathbb{R}^{N} .
$$

Thus there exists a non-increasing sequence of continuous functions $\gamma_{1}^{m}: \Omega \times \mathbb{R}^{N} \rightarrow[0,+\infty)$ such that

$$
\gamma_{1}(x, \lambda, \theta)=\inf _{m} \gamma_{1}^{m}(x, \theta)=\lim _{m} \gamma_{1}^{m}(x, \theta) \leqslant C|\theta|, \forall(x, \theta) \in \Omega \times \mathbb{R}^{N}
$$

Proposition 4.11. $\quad i) \quad W_{2}(x, A, 0,0) \leqslant W(x, A, 0), \forall(x, A) \in \Omega \times \mathbb{R}^{d \times N}$;

ii) for every $x \in \Omega$, every $A_{1}, A_{2} \in \mathbb{R}^{d \times N}$, and all $L, M_{1}, M_{2} \in \mathbb{R}^{d \times N \times N}$ we have that

$$
\left|W_{2}\left(x, A_{1}, L, M_{1}\right)-W_{2}\left(x, A_{2}, L, M_{2}\right)\right| \leqslant C\left(\left|A_{1}-A_{2}\right|+\left|M_{1}-M_{2}\right|\right) .
$$

Proof. The proof of $i)$ is immediate since the function $u=0$ is admissible for $W_{2}(x, A, 0,0)$.

To prove $i$ ) we will show that

$$
W_{2}\left(x, A_{1}, L, M_{1}\right) \leqslant W_{2}\left(x, A_{2}, L, M_{2}\right)+C\left(\left|A_{1}-A_{2}\right|+\left|M_{1}-M_{2}\right|\right),
$$

$\forall x \in \Omega, \forall A_{1}, A_{2} \in \mathbb{R}^{d \times N}, \forall L, M_{1}, M_{2} \in \mathbb{R}^{d \times N \times N}$; the reverse inequality follows by interchanging the roles of $A_{1}$ and $A_{2}$ and $M_{1}$ and $M_{2}$.

Fix $\varepsilon>0$ and let $u \in S B V\left(Q ; \mathbb{R}^{d \times N}\right)$ be such that $\left.u\right|_{\partial Q}(y)=L y, \int_{Q} \nabla u(y) d y=M_{2}$ and

$$
\int_{Q} W\left(x, A_{2}, \nabla u(y)\right) d y+\int_{S_{u} \cap Q} \Psi_{2}\left(x,[u(y)], \nu_{u}(y)\right) d \mathcal{H}^{N-1}(y) \leqslant W_{2}\left(x, A_{2}, L, M_{2}\right)+\varepsilon .
$$

By Lemma 2.5, let $v \in S B V\left(Q ; \mathbb{R}^{d \times N}\right)$ be such that $\left.v\right|_{\partial Q}=0, \nabla v=M_{1}-M_{2}$ a.e. in $Q$ and $\left|D^{s} v\right|(Q) \leqslant C\left|M_{1}-M_{2}\right|$, and define $w=u+v$. Then $w$ is admissible for $W_{2}\left(x, A_{1}, L, M_{1}\right)$ so by (H8), (H2) and (H5),

$$
\begin{aligned}
W_{2}\left(x, A_{1}, L, M_{1}\right) \leqslant & \int_{Q} W\left(x, A_{1}, \nabla w(y)\right) d y+\int_{S_{w} \cap Q} \Psi_{2}\left(x,[w(y)], \nu_{w}(y)\right) d \mathcal{H}^{N-1}(y) \\
\leqslant & \int_{Q} W\left(x, A_{1}, \nabla u(y)+M_{1}-M_{2}\right) d y \\
& +\int_{S_{u} \cap Q} \Psi_{2}\left(x,[u(y)], \nu_{u}(y)\right) d \mathcal{H}^{N-1}(y)+\int_{S_{v} \cap Q} \Psi_{2}\left(x,[v(y)], \nu_{v}(y)\right) d \mathcal{H}^{N-1}(y) \\
\leqslant & \int_{Q} W\left(x, A_{2}, \nabla u(y)\right) d y+C\left(\left|A_{1}-A_{2}\right|+\left|M_{1}-M_{2}\right|\right) \\
& +\int_{S_{u} \cap Q} \Psi_{2}\left(x,[u(y)], \nu_{u}(y)\right) d \mathcal{H}^{N-1}(y)+C\left|D^{s} v\right|(Q) \\
\leqslant & W_{2}\left(x, A_{2}, L, M_{2}\right)+\varepsilon+C\left(\left|A_{1}-A_{2}\right|+\left|M_{1}-M_{2}\right|\right),
\end{aligned}
$$

thus to conclude the desired inequality it suffices to let $\varepsilon \rightarrow 0^{+}$.

Proposition 4.12. $\quad i) \gamma_{2}(x, A, \Lambda, \nu) \leqslant C|\Lambda|, \forall(x, A, \Lambda, \nu) \in \Omega \times \mathbb{R}^{d \times N} \times \mathbb{R}^{d \times N} \times S^{N-1}$;

ii) for every $x_{0} \in \Omega$ and for every $\varepsilon>0$ there exists $\delta>0$ such that

$$
\begin{array}{r}
\left|x-x_{0}\right|<\delta \Rightarrow\left|\gamma_{2}\left(x_{0}, A, \Lambda, \nu\right)-\gamma_{2}(x, A, \Lambda, \nu)\right| \leqslant \varepsilon C(1+|\Lambda|), \\
\forall(x, A, \Lambda, \nu) \in \Omega \times \mathbb{R}^{d \times N} \times \mathbb{R}^{d \times N} \times S^{N-1} ;
\end{array}
$$


iii) for every $\left(x, A_{1}, \Lambda_{1}, \nu\right),\left(x, A_{2}, \Lambda_{2}, \nu\right) \in \Omega \times \mathbb{R}^{d \times N} \times \mathbb{R}^{d \times N} \times S^{N-1}$ we have that

$$
\left|\gamma_{2}\left(x, A_{1}, \Lambda_{1}, \nu\right)-\gamma_{2}\left(x, A_{2}, \Lambda_{2}, \nu\right)\right| \leqslant C\left(\left|A_{1}-A_{2}\right|+\left|\Lambda_{1}-\Lambda_{2}\right|\right),
$$

iv) $\gamma_{2}$ is upper semicontinuous in $\Omega \times \mathbb{R}^{d \times N} \times \mathbb{R}^{d \times N} \times S^{N-1}$.

Proof. The proof of $i$ ) follows immediately from the fact that the function $\gamma_{(\Lambda, \nu)}$ is admissible for $\gamma_{2}(x, A, \Lambda, \nu)$, from hypotheses (H5) and since $W^{\infty}(x, A, 0)=0$.

To prove $i$ ) fix $x_{0} \in \Omega$ and $\varepsilon>0$. By (3.4) and (H6) let $\delta>0$ be such that

$$
\left|x-x_{0}\right|<\delta \Rightarrow\left|W^{\infty}(x, A, M)-W^{\infty}\left(x_{0}, A, M\right)\right| \leqslant \varepsilon C|M|
$$

and

$$
\left|x-x_{0}\right|<\delta \Rightarrow\left|\Psi_{2}\left(x_{0}, \Lambda, \nu\right)-\Psi_{2}(x, \Lambda, \nu)\right| \leqslant \varepsilon C|\Lambda|
$$

Let $u_{n} \in S B V\left(Q_{\nu} ; \mathbb{R}^{d \times N}\right)$ be such that $\left.u_{n}\right|_{\partial Q_{\nu}}=\gamma_{(\Lambda, \nu)}, \int_{Q_{\nu}} \nabla u_{n}(y) d y=0$ and

$$
\int_{Q_{\nu}} W^{\infty}\left(x_{0}, A, \nabla u_{n}(y)\right) d y+\int_{S_{u_{n}} \cap Q_{\nu}} \Psi_{2}\left(x_{0},\left[u_{n}(y)\right], \nu_{u_{n}}(y)\right) d \mathcal{H}^{N-1}(y) \leqslant \gamma_{2}\left(x_{0}, A, \Lambda, \nu\right)+\frac{1}{n} .
$$

By (3.2), (H5) and $i$ ) we have

$$
\begin{aligned}
& \int_{Q_{\nu}}\left|\nabla u_{n}(y)\right| d y+\int_{S_{u_{n}} \cap Q_{\nu}}\left|\left[u_{n}(y)\right]\right| d \mathcal{H}^{N-1}(y) \\
& \leqslant C \int_{Q_{\nu}} W^{\infty}\left(x_{0}, A, \nabla u_{n}(y)\right) d y+C \int_{S_{u_{n}} \cap Q_{\nu}} \Psi_{2}\left(x_{0},\left[u_{n}(y)\right], \nu_{u_{n}}(y)\right) d \mathcal{H}^{N-1}(y) \\
& \leqslant C\left(\gamma_{2}\left(x_{0}, A, \Lambda, \nu\right)+\frac{1}{n}\right) \leqslant C(1+|\Lambda|) .
\end{aligned}
$$

Hence, if $\left|x-x_{0}\right|<\delta$, it follows by (4.22), (4.23) and 4.24) that

$$
\begin{aligned}
\gamma_{2}(x, A, \Lambda, \nu)-\gamma_{2}\left(x_{0}, A, \Lambda, \nu\right) & \leqslant \int_{Q_{\nu}} W^{\infty}\left(x, A, \nabla u_{n}(y)\right) d y+\int_{S_{u_{n}} \cap Q_{\nu}} \Psi_{2}\left(x,\left[u_{n}(y)\right], \nu_{u_{n}}(y)\right) d \mathcal{H}^{N-1}(y) \\
& -\int_{Q_{\nu}} W^{\infty}\left(x_{0}, A, \nabla u_{n}(y)\right) d y-\int_{S_{u_{n}} \cap Q_{\nu}} \Psi_{2}\left(x_{0},\left[u_{n}(y)\right], \nu_{u_{n}}(y)\right) d \mathcal{H}^{N-1}(y)+\frac{1}{n} \\
\leqslant & \int_{Q_{\nu}} \varepsilon C\left|\nabla u_{n}(y)\right| d y+\int_{S_{u_{n}} \cap Q_{\nu}} \varepsilon C\left|\left[u_{n}(y)\right]\right| d \mathcal{H}^{N-1}(y)+\frac{1}{n} \\
\leqslant & \varepsilon C(1+|\Lambda|)+\frac{1}{n} .
\end{aligned}
$$

Letting $n \rightarrow+\infty$ we conclude that

$$
\gamma_{2}(x, A, \Lambda, \nu)-\gamma_{2}\left(x_{0}, A, \Lambda, \nu\right) \leqslant \varepsilon C(1+|\Lambda|) .
$$

Changing the roles of $x$ and $x_{0}$ we obtain the result.

We now prove $i i i)$. Let $u \in S B V\left(Q_{\nu} ; \mathbb{R}^{d \times N}\right)$ be such that $\left.u\right|_{\partial Q_{\nu}}=\gamma_{\left(\Lambda_{1}, \nu\right)}, \int_{Q_{\nu}} \nabla u(y) d y=0$ and

$$
\int_{Q_{\nu}} W^{\infty}\left(x, A_{1}, \nabla u(y)\right) d y+\int_{S_{u} \cap Q_{\nu}} \Psi_{2}\left(x,[u(y)], \nu_{u}(y)\right) d \mathcal{H}^{N-1}(y) \leqslant \gamma_{2}\left(x, A_{1}, \Lambda_{1}, \nu\right)+\varepsilon .
$$


Let $v=\gamma_{\left(\Lambda_{2}, \nu\right)}-\gamma_{\left(\Lambda_{1}, \nu\right)}$ and define $w=u+v$. Since $w$ is admissible for $\gamma_{2}\left(x, A_{2}, \Lambda_{2}, \nu\right)$ we have by (3.3), (H8) and (H5),

$$
\begin{aligned}
\gamma_{2}\left(x, A_{2}, \Lambda_{2}, \nu\right) \leqslant & \int_{Q_{\nu}} W^{\infty}\left(x, A_{2}, \nabla w(y)\right) d y+\int_{S_{w} \cap Q_{\nu}} \Psi_{2}\left(x,[w(y)], \nu_{w}(y)\right) d \mathcal{H}^{N-1}(y) \\
\leqslant & \int_{Q_{\nu}} W^{\infty}\left(x, A_{1}, \nabla u(y)\right) d y+\int_{S_{u} \cap Q_{\nu}} \Psi_{2}\left(x,[u(y)], \nu_{u}(y)\right) d \mathcal{H}^{N-1}(y) \\
& +\int_{S_{v} \cap Q_{\nu}} \Psi_{2}\left(x,[v(y)], \nu_{v}(y)\right) d \mathcal{H}^{N-1}(y) \\
\leqslant & \gamma_{2}\left(x, A_{1}, \Lambda_{1}, \nu\right)+\varepsilon+\int_{\left\{y \in Q_{\nu}: y \cdot \nu=0\right\}} \Psi_{2}\left(x, \Lambda_{2}-\Lambda_{1}, \nu\right) d \mathcal{H}^{N-1}(y) \\
\leqslant & \gamma_{2}\left(x, A_{1}, \Lambda_{1}, \nu\right)+\varepsilon+C\left|\Lambda_{2}-\Lambda_{1}\right|,
\end{aligned}
$$

so to prove the first inequality it suffices to let $\varepsilon \rightarrow 0^{+}$. The other inequality is obtained in a similar fashion.

To prove $i v)$, due to the result of $i i i)$ it suffices to show that $(x, \nu) \rightarrow \gamma_{2}(x, A, \Lambda, \nu)$ is upper semicontinuous, for every $A, \Lambda \in \mathbb{R}^{d \times N}$. By a change of variables argument, choosing a rotation $R$ such that $R e_{N}=\nu$, it is easy to see that

$$
\begin{aligned}
\gamma_{2}(x, A, \Lambda, \nu)=\inf _{u \in S B V\left(Q ; \mathbb{R}^{d \times N}\right)}\{ & \int_{Q} W^{\infty}\left(x, A, \nabla u(y) R^{T}\right) d y \\
+ & \int_{S_{u} \cap Q} \Psi_{2}\left(x,[u(y)], \nu_{u}(y)\right) d \mathcal{H}^{N-1}(y):\left.u\right|_{\partial Q}=\gamma_{\left(\Lambda, e_{N}\right)}, \\
& \left.\int_{Q} \nabla u(y) d y=0, R e_{N}=\nu, R \in S O(N)\right\} .
\end{aligned}
$$

Let $\left(x_{n}, \nu_{n}\right) \rightarrow(x, \nu)$. Given $\varepsilon>0$, let $u_{\varepsilon} \in S B V\left(Q ; \mathbb{R}^{d \times N}\right)$ be such that $\left.u_{\varepsilon}\right|_{\partial Q}=\gamma_{\left(\Lambda, e_{N}\right)}$, $\int_{Q} \nabla u_{\varepsilon}(y) d y=0$ and

$$
\left|\gamma_{2}(x, A, \Lambda, \nu)-\int_{Q} W^{\infty}\left(x, A, \nabla u_{\varepsilon}(y) R^{T}\right) d y-\int_{S_{u_{\varepsilon}} \cap Q} \Psi_{2}\left(x,\left[u_{\varepsilon}(y)\right], \nu_{u_{\varepsilon}}(y)\right) d \mathcal{H}^{N-1}(y)\right|<\varepsilon .
$$

Let $K$ be a compact subset of $\Omega$ containing a neighborhood of $x$ and choose $\delta>0$ such that (3.4) and (H6) are satisfied uniformly in $K$, i.e.

$$
y, y^{\prime} \in K,\left|y-y^{\prime}\right|<\delta \Rightarrow\left|W^{\infty}(y, A, M)-W^{\infty}\left(y^{\prime}, A, M\right)\right| \leqslant \varepsilon C|M|,
$$

for every $(A, M) \in \mathbb{R}^{d \times N} \times \mathbb{R}^{d \times N \times N}$, and

$$
y, y^{\prime} \in K,\left|y-y^{\prime}\right|<\delta \Rightarrow\left|\Psi_{2}(y, \Lambda, \nu)-\Psi_{2}\left(y^{\prime}, \Lambda, \nu\right)\right| \leqslant \varepsilon C|\Lambda|,
$$

for all $(\Lambda, \nu) \in \mathbb{R}^{d \times N} \times S^{N-1}$. Choosing rotations $R_{n}$ such that $R_{n} e_{N}=\nu_{n}, R_{n} \rightarrow R$, by (4.27), (4.28), (3.3), (3.2), (H5) and (4.26) we have that

$$
\begin{aligned}
& \mid \int_{Q} W^{\infty}\left(x, A, \nabla u_{\varepsilon}(y) R^{T}\right) d y+\int_{S_{u_{\varepsilon}} \cap Q} \Psi_{2}\left(x,\left[u_{\varepsilon}(y)\right], \nu_{u_{\varepsilon}}(y)\right) d \mathcal{H}^{N-1}(y) \\
& \quad-\int_{Q} W^{\infty}\left(x_{n}, A, \nabla u_{\varepsilon}(y) R_{n}^{T}\right) d y-\int_{S_{u_{\varepsilon}} \cap Q} \Psi_{2}\left(x_{n},\left[u_{\varepsilon}(y)\right], \nu_{u_{\varepsilon}}(y)\right) d \mathcal{H}^{N-1}(y) \mid \\
& \leqslant \int_{Q}\left|W^{\infty}\left(x, A, \nabla u_{\varepsilon}(y) R^{T}\right)-W^{\infty}\left(x_{n}, A, \nabla u_{\varepsilon}(y) R^{T}\right)\right| d y \\
& \quad+\int_{Q}\left|W^{\infty}\left(x_{n}, A, \nabla u_{\varepsilon}(y) R^{T}\right)-W^{\infty}\left(x_{n}, A, \nabla u_{\varepsilon}(y) R_{n}^{T}\right)\right| d y
\end{aligned}
$$




$$
\begin{aligned}
& +\int_{S_{u_{\varepsilon}} \cap Q} \varepsilon C\left|\left[u_{\varepsilon}(y)\right]\right| d \mathcal{H}^{N-1}(y) \\
\leqslant & \int_{Q} \varepsilon C\left|\nabla u_{\varepsilon}(y) R^{T}\right| d y+\int_{Q} C\left|\nabla u_{\varepsilon}(y)\right|\left|R_{n}^{T}-R^{T}\right| d y+\int_{S_{u_{\varepsilon}} \cap Q} \varepsilon C\left|\left[u_{\varepsilon}(y)\right]\right| d \mathcal{H}^{N-1}(y) \\
\leqslant & \varepsilon C \int_{Q} W^{\infty}\left(x, A, \nabla u_{\varepsilon}(y) R^{T}\right) d y+\varepsilon C \int_{S_{u_{\varepsilon}} \cap Q} \Psi_{2}\left(x,\left[u_{\varepsilon}(y)\right], \nu_{u_{\varepsilon}}(y)\right) d \mathcal{H}^{N-1}(y) \\
& +\left|R_{n}^{T}-R^{T}\right| \int_{Q} W^{\infty}\left(x, A, \nabla u_{\varepsilon}(y) R^{T}\right) d y \\
\leqslant & \left(\varepsilon C+\left|R_{n}^{T}-R^{T}\right|\right)\left(\varepsilon+\gamma_{2}(x, A, \Lambda, \nu)\right)=O(\varepsilon)+O\left(\left|R_{n}^{T}-R^{T}\right|\right) .
\end{aligned}
$$

Thus, by 4.25 and 4.26),

$$
\begin{aligned}
\gamma_{2}\left(x_{n}, A, \Lambda, \nu_{n}\right) \leqslant & \int_{Q} W^{\infty}\left(x_{n}, A, \nabla u_{\varepsilon}(y) R_{n}^{T}\right) d y+\int_{S_{u_{\varepsilon}} \cap Q} \Psi_{2}\left(x_{n},\left[u_{\varepsilon}(y)\right], \nu_{u_{\varepsilon}}(y)\right) d \mathcal{H}^{N-1}(y) \\
\leqslant & \int_{Q} W^{\infty}\left(x, A, \nabla u_{\varepsilon}(y) R^{T}\right) d y+\int_{S_{u_{\varepsilon}} \cap Q} \Psi_{2}\left(x,\left[u_{\varepsilon}(y)\right], \nu_{u_{\varepsilon}}(y)\right) d \mathcal{H}^{N-1}(y) \\
& +O(\varepsilon)+O\left(\left|R_{n}^{T}-R^{T}\right|\right) \\
\leqslant & O(\varepsilon)+O\left(\left|R_{n}^{T}-R^{T}\right|\right)+\gamma_{2}(x, A, \Lambda, \nu)
\end{aligned}
$$

Therefore, letting $\varepsilon \rightarrow 0^{+}$, and passing to the limit as $n \rightarrow+\infty$, since $R_{n} \rightarrow R$, we conclude that

$$
\limsup _{n \rightarrow+\infty} \gamma_{2}\left(x_{n}, A, \Lambda, \nu_{n}\right) \leqslant \gamma_{2}(x, A, \Lambda, \nu)
$$

Remark 4.13. $\gamma_{2}(x, A, \Lambda, \nu)$ can be extended to $\Omega \times \mathbb{R}^{d \times N} \times \mathbb{R}^{d \times N} \times \mathbb{R}^{N}$ as a positively homogeneous of degree one function in the fourth variable in the following way

$$
\gamma_{2}(x, A, \Lambda, \theta)= \begin{cases}|\theta| \gamma_{2}\left(x, A, \Lambda, \frac{\theta}{|\theta|}\right), & \text { if } \theta \in \mathbb{R}^{N} \backslash\{0\} \\ 0, & \text { if } \theta=0\end{cases}
$$

By Proposition 4.12 this extension is upper semicontinuous in $\Omega \times \mathbb{R}^{d \times N} \times \mathbb{R}^{d \times N} \times \mathbb{R}^{N}$ and satisfies

$$
\gamma_{2}(x, A, \Lambda, \theta) \leqslant C|\Lambda||\theta|, \forall(x, A, \Lambda, \theta) \in \Omega \times \mathbb{R}^{d \times N} \times \mathbb{R}^{d \times N} \times \mathbb{R}^{N} .
$$

Thus there exists a non-increasing sequence of continuous functions $\gamma_{2}^{m}: \Omega \times \mathbb{R}^{N} \rightarrow[0,+\infty)$ such that

$$
\gamma_{2}(x, A, \Lambda, \theta)=\inf _{m} \gamma_{2}^{m}(x, \theta)=\lim _{m} \gamma_{2}^{m}(x, \theta) \leqslant C|\theta|, \forall(x, \theta) \in \Omega \times \mathbb{R}^{N}
$$

\section{INTEGRAL REPRESENTATION OF $I(g, G, \Gamma)$}

The proof of the integral representation of $I$ follows along the lines of the proofs in [12] (for $I_{2}$ ) and in [7] (for $I_{1}$ ), together with arguments in [9] in order to deal with the explicit dependence on the position variable $x$. In what follows, we mostly restrict our attention to the integral representation of $I_{1}$ since that of $I_{2}$ can be derived in a similar manner.

5.1. Integral representation of $I_{1}(g, G, \Gamma)$. In this section we will prove the following result.

Theorem 5.1. For all $(g, G, \Gamma) \in S D^{2}\left(\Omega ; \mathbb{R}^{d}\right)$, under hypotheses (H1)-(H8), we have that

$$
I_{1}(g, G, \Gamma)=\int_{\Omega} W_{1}(x, G(x)-\nabla g(x)) d x+\int_{S_{g} \cap \Omega} \gamma_{1}\left(x,[g(x)], \nu_{g}(x)\right) d \mathcal{H}^{N-1}(x) .
$$


5.1.1. The lower bound inequality. We begin by obtaining a lower bound for $I_{1}(g, G, \Gamma)$.

Proposition 5.2. For all $(g, G, \Gamma) \in S D^{2}\left(\Omega ; \mathbb{R}^{d}\right)$, under hypotheses (H1) - (H8), we have that

$$
I_{1}(g, G, \Gamma) \geqslant \int_{\Omega} W_{1}(x, G(x)-\nabla g(x)) d x+\int_{S_{g} \cap \Omega} \gamma_{1}\left(x,[g(x)], \nu_{g}(x)\right) d \mathcal{H}^{N-1}(x) .
$$

Proof. Let $\left\{u_{n}\right\} \subset S B V^{2}\left(\Omega ; \mathbb{R}^{d}\right)$ be an admissible sequence for $I_{1}(g, G, \Gamma)$ such that

$$
\lim _{n \rightarrow+\infty} \int_{S_{u_{n}} \cap \Omega} \Psi_{1}\left(x,\left[u_{n}\right](x), \nu_{u_{n}}(x)\right) d \mathcal{H}^{N-1}(x)<+\infty .
$$

For each Borel set $B \subset \bar{\Omega}$ define the sequence of Radon measures $\left\{\mu_{n}\right\}$ by

$$
\mu_{n}(B):=\int_{S_{u_{n} \cap B}} \Psi_{1}\left(x,\left[u_{n}\right](x), \nu_{u_{n}}(x)\right) d \mathcal{H}^{N-1}(x) .
$$

By the choice of $u_{n}$, the sequence $\left\{\mu_{n}\right\}$ is bounded so there exists $\mu \in \mathcal{M}^{+}(\Omega)$ such that, up to a subsequence (not relabeled), $\mu_{n} \stackrel{*}{\rightarrow} \mu$ in the sense of measures. By the Radon-Nikodym theorem we may decompose $\mu$ as the sum of three mutually singular non-negative measures

$$
\mu=\mu_{a} \mathcal{L}^{N}+\mu_{j} \mathcal{H}^{N-1}\left\lfloor S_{g}+\mu_{s} .\right.
$$

Using the blow-up method it suffices to show that, for $\mathcal{L}^{N}$ a.e. $x_{0} \in \Omega$,

$$
\mu_{a}\left(x_{0}\right)=\frac{d \mu}{d \mathcal{L}^{N}}\left(x_{0}\right) \geqslant W_{1}\left(x_{0}, G\left(x_{0}\right)-\nabla g\left(x_{0}\right)\right),
$$

and, for $\mathcal{H}^{N-1}$ a.e. $x_{0} \in S_{g} \cap \Omega$,

$$
\mu_{j}\left(x_{0}\right)=\frac{d \mu}{d \mathcal{H}^{N-1}\left\lfloor S_{g}\right.}\left(x_{0}\right) \geqslant \gamma_{1}\left(x_{0},\left[g\left(x_{0}\right)\right], \nu_{g}\left(x_{0}\right)\right) .
$$

Assuming (5.1) and (5.2) hold, we then obtain

$$
\begin{aligned}
\lim _{n \rightarrow+\infty} & \int_{S_{u_{n}} \cap \Omega} \Psi_{1}\left(x,\left[u_{n}\right](x), \nu_{u_{n}}(x)\right) d \mathcal{H}^{N-1}(x) \\
& \geqslant \int_{\Omega} \mu_{a}(x) d x+\int_{S_{g} \cap \Omega} \mu_{j}(x) d \mathcal{H}^{N-1}(x) \\
& \geqslant \int_{\Omega} W_{1}(x, G(x)-\nabla g(x)) d x+\int_{S_{g} \cap \Omega} \gamma_{1}\left(x,[g(x)], \nu_{g}(x)\right) d \mathcal{H}^{N-1}(x),
\end{aligned}
$$

and the result follows by taking the infimum over all sequences $\left\{u_{n}\right\}$ satisfying the above properties.

The remainder of this section will be devoted to the proofs of inequalities (5.1) and (5.2).

Proposition 5.3. For $\mathcal{L}^{N}$ a.e. $x_{0} \in \Omega$ the following inequality holds,

$$
\frac{d \mu}{d \mathcal{L}^{N}}\left(x_{0}\right) \geqslant W_{1}\left(x_{0}, G\left(x_{0}\right)-\nabla g\left(x_{0}\right)\right) .
$$

Proof. Let $x_{0} \in \Omega$ be a point of approximate differentiability of $g$ and of approximate continuity of $G$. Moreover, $x_{0}$ is chosen so that $\frac{d \mu}{d \mathcal{L}^{N}}\left(x_{0}\right)$ exists and is finite. Let $\left\{\delta_{k}\right\}$ be a sequence of positive real numbers such that $\delta_{k} \rightarrow 0^{+}$and $\mu\left(\partial Q\left(x_{0}, \delta_{k}\right)\right)=0$. Therefore,

$$
\lim _{n \rightarrow+\infty} \mu_{n}\left(Q\left(x_{0}, \delta_{k}\right)\right)=\mu\left(Q\left(x_{0}, \delta_{k}\right)\right),
$$

and so

$$
\begin{aligned}
\frac{d \mu}{d \mathcal{L}^{N}}\left(x_{0}\right) & =\lim _{k \rightarrow+\infty} \frac{\mu\left(Q\left(x_{0}, \delta_{k}\right)\right)}{\mathcal{L}^{N}\left(Q\left(x_{0}, \delta_{k}\right)\right)} \\
& =\lim _{k, n} \frac{1}{\delta_{k}^{N}} \int_{S_{u_{n}} \cap Q\left(x_{0}, \delta_{k}\right)} \Psi_{1}\left(x,\left[u_{n}(x)\right], \nu_{u_{n}}(x)\right) d \mathcal{H}^{N-1}(x) \\
& =\lim _{k, n} \frac{1}{\delta_{k}} \int_{Q \cap\left\{y: x_{0}+\delta_{k} y \in S_{u_{n}}\right\}} \Psi_{1}\left(x_{0}+\delta_{k} y,\left[u_{n}\left(x_{0}+\delta_{k} y\right)\right], \nu_{u_{n}}\left(x_{0}+\delta_{k} y\right)\right) d \mathcal{H}^{N-1}(y) .
\end{aligned}
$$


For $y \in Q$ define

$$
v_{n, k}(y):=\frac{u_{n}\left(x_{0}+\delta_{k} y\right)-g\left(x_{0}\right)}{\delta_{k}} \text { and } v_{0}(y):=\nabla g\left(x_{0}\right) y
$$

Notice that, as $x_{0}$ is a point of approximate differentiability of $g$ and of approximate continuity of $G$,

$$
v_{n, k} \underset{k, n \rightarrow+\infty}{\stackrel{L^{1}}{\longrightarrow}} v_{0} \text { and } \nabla v_{k, n} \underset{k, n \rightarrow+\infty}{\stackrel{L^{1}}{\rightarrow}} G\left(x_{0}\right) .
$$

Then, by (H7), (H6) and for $k$ large enough, we have

$$
\begin{aligned}
\frac{d \mu}{d \mathcal{L}^{N}}\left(x_{0}\right) & =\lim _{k, n} \int_{Q \cap S_{v_{n, k}}} \Psi_{1}\left(x_{0}+\delta_{k} y,\left[v_{n, k}(y)\right], \nu_{v_{n, k}}(y)\right) d \mathcal{H}^{N-1}(y) \\
& \geqslant \lim _{k, n} \int_{Q \cap S_{v_{n, k}}} \Psi_{1}\left(x_{0},\left[v_{n, k}(y)\right], \nu_{v_{n, k}}(y)\right) d \mathcal{H}^{N-1}(y)-\varepsilon C\left|D^{s} v_{n, k}\right|(Q) \\
& \geqslant \lim _{k, n} \int_{Q \cap S_{v_{n, k}}} \Psi_{1}\left(x_{0},\left[v_{n, k}(y)\right], \nu_{v_{n, k}}(y)\right) d \mathcal{H}^{N-1}(y)+O(\varepsilon),
\end{aligned}
$$

where we have also used (H5) and (5.3). We must now modify $\left\{v_{n, k}\right\}$ in order to obtain a new sequence which is zero on the boundary of $Q$ and whose gradient equals $G\left(x_{0}\right)-\nabla g\left(x_{0}\right)$. For $y \in Q$, define $w_{n, k}(y):=v_{n, k}(y)-v_{0}(y)$. Since $w_{n, k} \underset{k, n \rightarrow+\infty}{\stackrel{L^{1}}{\rightarrow}} 0$, we may choose $\left.r_{n, k} \in\right] 0,1[$ such that $r_{n, k} \underset{k, n \rightarrow+\infty}{\longrightarrow} 1$ and

$$
\lim _{k, n} \int_{\partial Q\left(0, r_{n, k}\right)}\left|w_{n, k}(y)\right| d \mathcal{H}^{N-1}(y)=0 .
$$

By Theorem 2.4, let $\rho_{n, k} \in S B V\left(Q ; \mathbb{R}^{d}\right)$ be such that $\nabla \rho_{n, k}(y)=G\left(x_{0}\right)-\nabla v_{n, k}(y)$,

$$
\left\|D^{s} \rho_{n, k}\right\|\left(Q\left(0, r_{n, k}\right)\right) \leqslant C\left\|G\left(x_{0}\right)-\nabla v_{n, k}\right\|_{L^{1}},
$$

and define $z_{n, k}:=w_{n, k}+\rho_{n, k}$ for $y \in Q\left(0, r_{n, k}\right)$. Notice that $\nabla z_{n, k}(y)=G\left(x_{0}\right)-\nabla g\left(x_{0}\right)$. Also, by (5.4), $\nabla \rho_{n, k} \underset{k, n \rightarrow+\infty}{\stackrel{L^{1}}{\rightarrow}} 0$, so $\left\|D^{s} \rho_{n, k}\right\|\left(Q\left(0, r_{n, k}\right)\right) \rightarrow 0$. Thus, by the continuity of the trace operator with respect to the intermediate topology it follows that

$$
\lim _{k, n} \int_{\partial Q\left(0, r_{n, k}\right)}\left|\rho_{n, k}(y)\right| d \mathcal{H}^{N-1}(y)=0 .
$$

We now apply Lemma 2.5 in order to obtain a sequence $\left\{\eta_{n, k}\right\} \subset S B V\left(Q \backslash Q\left(0, r_{n, k}\right) ; \mathbb{R}^{d}\right)$ such that $\nabla \eta_{n, k}(y)=G\left(x_{0}\right)-\nabla g\left(x_{0}\right)$, for $\mathcal{L}^{N}$ a.e. $y \in Q \backslash Q\left(0, r_{n, k}\right), \eta_{n, k}=0$ on $\partial\left(Q \backslash Q\left(0, r_{n, k}\right)\right)$ and $\left\|D^{s} \eta_{n, k}\right\|\left(Q \backslash Q\left(0, r_{n, k}\right)\right) \leqslant C\left|Q \backslash Q\left(0, r_{n, k}\right)\right|$. Then the sequence

$$
\tilde{z}_{n, k}(y):= \begin{cases}z_{n, k}(y), & \text { if } y \in Q\left(0, r_{n, k}\right) \\ \eta_{n, k}(y), & \text { if } y \in Q \backslash Q\left(0, r_{n, k}\right)\end{cases}
$$

is admissible for $W_{1}\left(x, G\left(x_{0}\right)-\nabla g\left(x_{0}\right)\right)$ and satisfies, by (H5) and (H8),

$$
\begin{aligned}
& \int_{Q \cap \tilde{z}_{n, k}} \Psi_{1}\left(x_{0},\left[\tilde{z}_{n, k}(y)\right], \nu_{\tilde{z}_{n, k}}(y)\right) d \mathcal{H}^{N-1}(y) \\
& \leqslant \int_{Q\left(0, r_{n, k}\right) \cap S_{w_{n, k}}} \Psi_{1}\left(x_{0},\left[w_{n, k}(y)\right], \nu_{w_{n, k}}(y)\right) d \mathcal{H}^{N-1}(y) \\
& +\int_{Q\left(0, r_{n, k}\right) \cap S_{\rho_{n, k}}} \Psi_{1}\left(x_{0},\left[\rho_{n, k}(y)\right], \nu_{\rho_{n, k}}(y)\right) d \mathcal{H}^{N-1}(y) \\
& +C \int_{\partial Q\left(0, r_{n, k}\right)}\left|z_{n, k}(y)\right| d \mathcal{H}^{N-1}(y)+C \int_{\left[Q \backslash Q\left(0, r_{n, k}\right)\right] \cap S_{\eta_{n, k}}}\left|\left[\eta_{n, k}(y)\right]\right| d \mathcal{H}^{N-1}(y)
\end{aligned}
$$


SECOND-ORDER STRUCTURED DEFORMATIONS: RELAXATION, INTEGRAL REPRESENTATION AND APPLICATION\&1

$$
\begin{aligned}
& \leqslant \int_{Q \cap S_{v_{n, k}}} \Psi_{1}\left(x_{0},\left[v_{n, k}(y)\right], \nu_{v_{n, k}}(y)\right) d \mathcal{H}^{N-1}(y) \\
& +C \int_{Q\left(0, r_{n, k}\right) \cap S_{\rho_{n, k}}}\left|\left[\rho_{n, k}(y)\right]\right| d \mathcal{H}^{N-1}(y)+C \int_{\partial Q\left(0, r_{n, k}\right)}\left|w_{n, k}(y)\right| d \mathcal{H}^{N-1}(y) \\
& +C \int_{\partial Q\left(0, r_{n, k}\right)}\left|\rho_{n, k}(y)\right| d \mathcal{H}^{N-1}(y)+C \int_{\left[Q \backslash Q\left(0, r_{n, k}\right)\right] \cap S_{\eta_{n, k}}}\left|\left[\eta_{n, k}(y)\right]\right| d \mathcal{H}^{N-1}(y) .
\end{aligned}
$$

Since the last four integrals in the above expression converge to zero as $k, n \rightarrow+\infty$ we conclude from 5.5 that

$$
\begin{aligned}
\frac{d \mu}{d \mathcal{L}^{N}}\left(x_{0}\right) & \geqslant \liminf _{k, n} \int_{Q \cap S_{\tilde{z}_{n, k}}} \Psi_{1}\left(x_{0},\left[\tilde{z}_{n, k}(y)\right], \nu_{\tilde{z}_{n, k}}(y)\right) d \mathcal{H}^{N-1}(y)+O(\varepsilon) \\
& \geqslant W_{1}\left(x_{0}, G\left(x_{0}\right)-\nabla g\left(x_{0}\right)\right)+O(\varepsilon)
\end{aligned}
$$

so to conclude the result it suffices to let $\varepsilon \rightarrow 0^{+}$.

We proceed with the proof of (5.2).

Proposition 5.4. For $\mathcal{H}^{N-1}$ a.e. $x_{0} \in S_{g} \cap \Omega$ we have that

$$
\frac{d \mu}{d \mathcal{H}^{N-1}\left\lfloor S_{g}\right.}\left(x_{0}\right) \geqslant \gamma_{1}\left(x_{0},\left[g\left(x_{0}\right)\right], \nu_{g}\left(x_{0}\right)\right) \text {. }
$$

Proof. Let $x_{0} \in S_{g}$ be such that $\frac{d \mu}{d \mathcal{H}^{N-1}\left\lfloor S_{g}\right.}\left(x_{0}\right)$ exists and is finite, denote by $\nu:=\nu_{g}\left(x_{0}\right)$ and assume the point $x_{0}$ also satisfies

$$
\lim _{\delta \rightarrow 0^{+}} \frac{\mathcal{H}^{N-1}\left(S_{g} \cap Q_{\nu}\left(x_{0}, \delta\right)\right)}{\delta^{N-1}}=1
$$

and

$$
\lim _{\delta \rightarrow 0^{+}} \frac{1}{\delta^{N-1}} \int_{Q_{\nu}\left(x_{0}, \delta\right)}|G(x)| d x=0 .
$$

We point out that these conditions hold for $\mathcal{H}^{N-1}$ a.e. $x_{0} \in S_{g}$. Let $\left\{\delta_{k}\right\}$ be a sequence of positive real numbers such that $\delta_{k} \rightarrow 0^{+}$and $\mu\left(\partial Q_{\nu}\left(x_{0}, \delta_{k}\right)\right)=0$. Therefore,

$$
\lim _{n \rightarrow+\infty} \mu_{n}\left(Q_{\nu}\left(x_{0}, \delta_{k}\right)\right)=\mu\left(Q_{\nu}\left(x_{0}, \delta_{k}\right)\right)
$$

and so, by (5.6), (H6) and for $k$ large enough, we have

$$
\begin{aligned}
& \quad \frac{d \mu}{d \mathcal{H}^{N-1}\left\lfloor S_{g}\right.}\left(x_{0}\right)=\lim _{k, n} \frac{1}{\mathcal{H}^{N-1}\left(S_{g} \cap Q_{\nu}\left(x_{0}, \delta_{k}\right)\right)} \mu_{n}\left(Q_{\nu}\left(x_{0}, \delta_{k}\right)\right) \\
& =\lim _{k, n} \frac{1}{\mathcal{H}^{N-1}\left(S_{g} \cap Q_{\nu}\left(x_{0}, \delta_{k}\right)\right)} \int_{S_{u_{n}} \cap Q_{\nu}\left(x_{0}, \delta_{k}\right)} \Psi_{1}\left(x,\left[u_{n}(x)\right], \nu_{u_{n}}(x)\right) d \mathcal{H}^{N-1}(x) \\
& =\lim _{k, n} \frac{\delta_{k}^{N-1}}{\mathcal{H}^{N-1}\left(S_{g} \cap Q_{\nu}\left(x_{0}, \delta_{k}\right)\right)} \\
& \qquad \int_{Q_{\nu} \cap\left\{y: x_{0}+\delta_{k} y \in S_{u_{n}}\right\}} \Psi_{1}\left(x_{0}+\delta_{k} y,\left[u_{n}\left(x_{0}+\delta_{k} y\right)\right], \nu_{u_{n}}\left(x_{0}+\delta_{k} y\right)\right) d \mathcal{H}^{N-1}(y) \\
& \geqslant \\
& \geqslant \lim _{k, n} \int_{Q_{\nu} \cap\left\{y: x_{0}+\delta_{k} y \in S_{u_{n}}\right\}} \int_{Q_{\nu} \cap S_{w_{n, k}}} \Psi_{1}\left(x_{0},\left[w_{n, k}(y)\right], \nu_{w_{n, k}}(y)\right) d \mathcal{H}^{N-1}(y)-O(\varepsilon),
\end{aligned}
$$

where, for $y \in Q_{\nu}$, we define

$$
w_{n, k}(y):=u_{n}\left(x_{0}+\delta_{k} y\right)-g^{-}\left(x_{0}\right) .
$$


By definition of $g^{-}\left(x_{0}\right), g^{+}\left(x_{0}\right)$, by (5.7), and since $u_{n} \rightarrow g$ in $L^{1}\left(\Omega ; \mathbb{R}^{d}\right)$, and $\nabla u_{n} \rightarrow G$ in $L^{1}\left(\Omega ; \mathbb{R}^{d \times N}\right)$, one has

$$
w_{n, k} \underset{k, n \rightarrow+\infty}{\stackrel{L^{1}}{\longrightarrow}} \gamma_{\left(\left[g\left(x_{0}\right)\right], \nu\right)} \text { and } \nabla w_{k, n} \underset{k, n \rightarrow+\infty}{\stackrel{L^{1}}{\longrightarrow}} 0 .
$$

We must now modify $\left\{w_{n, k}\right\}$ in order to obtain a new sequence which is equal to $\gamma_{\left(\left[g\left(x_{0}\right)\right], \nu\right)}$ on the boundary of $Q_{\nu}$ and whose gradient is zero a.e. in $Q_{\nu}$. For $y \in Q_{\nu}$, define

$$
v_{n, k}(y):=w_{n, k}(y)-\gamma_{\left(\left[g\left(x_{0}\right)\right], \nu\right)}(y) .
$$

Since $v_{n, k} \underset{k, n \rightarrow+\infty}{\stackrel{L^{1}}{\longrightarrow}} 0$, we may choose $\left.r_{n, k} \in\right] 0,1\left[\right.$ such that $r_{n, k} \underset{k, n \rightarrow+\infty}{\longrightarrow} 1$ and

$$
\lim _{k, n} \int_{\partial Q_{\nu}\left(0, r_{n, k}\right)}\left|v_{n, k}(y)\right| d \mathcal{H}^{N-1}(y)=0 .
$$

By Theorem 2.4. let $\rho_{n, k} \in S B V\left(Q_{\nu} ; \mathbb{R}^{d}\right)$ be such that $\nabla \rho_{n, k}(y)=-\nabla w_{n, k}(y)$,

$$
\left\|D^{s} \rho_{n, k}\right\|\left(Q_{\nu}\left(0, r_{n, k}\right)\right) \leqslant C\left\|\nabla w_{n, k}\right\|_{L^{1}}
$$

and define $z_{n, k}:=w_{n, k}+\rho_{n, k}$ for $y \in Q_{\nu}\left(0, r_{n, k}\right)$. Notice that $\nabla z_{n, k}(y)=0$ in $Q_{\nu}\left(0, r_{n, k}\right)$. Also, by (5.9), $\nabla \rho_{n, k} \underset{k, n \rightarrow+\infty}{\stackrel{L^{1}}{\longrightarrow}} 0$, so $\left\|D^{s} \rho_{n, k}\right\|\left(Q_{\nu}\left(0, r_{n, k}\right)\right) \rightarrow 0$. Thus, by the continuity of the trace operator with respect to the intermediate topology it follows that

$$
\lim _{k, n} \int_{\partial Q_{\nu}\left(0, r_{n, k}\right)}\left|\rho_{n, k}(y)\right| d \mathcal{H}^{N-1}(y)=0 .
$$

Then the sequence

$$
\tilde{z}_{n, k}(y):= \begin{cases}z_{n, k}(y), & \text { if } y \in Q_{\nu}\left(0, r_{n, k}\right) \\ \gamma_{\left(\left[g\left(x_{0}\right)\right], \nu\right)}(y), & \text { if } y \in Q_{\nu} \backslash Q_{\nu}\left(0, r_{n, k}\right)\end{cases}
$$

is admissible for $\gamma_{1}\left(x_{0},\left[g\left(x_{0}\right)\right], \nu\right)$ and satisfies, by (H5) and (H8),

$$
\begin{aligned}
& \int_{Q_{\nu} \cap S_{z_{n, k}}} \Psi_{1}\left(x_{0},\left[\tilde{z}_{n, k}(y)\right], \nu_{\tilde{z}_{n, k}}(y)\right) d \mathcal{H}^{N-1}(y) \\
& \leqslant \int_{Q_{\nu}\left(0, r_{n, k}\right) \cap S_{w_{n, k}}} \Psi_{1}\left(x_{0},\left[w_{n, k}(y)\right], \nu_{w_{n, k}}(y)\right) d \mathcal{H}^{N-1}(y) \\
& +\int_{Q\left(0, r_{n, k}\right) \cap S_{\rho_{n, k}}} \Psi_{1}\left(x_{0},\left[\rho_{n, k}(y)\right], \nu_{\rho_{n, k}}(y)\right) d \mathcal{H}^{N-1}(y) \\
& +C \int_{\partial Q_{\nu}\left(0, r_{n, k}\right)}\left|z_{n, k}(y)-\gamma_{\left(\left[g\left(x_{0}\right)\right], \nu\right)}(y)\right| d \mathcal{H}^{N-1}(y) \\
& +C \int_{\left[Q_{\nu} \backslash Q_{\nu}\left(0, r_{n, k}\right)\right] \cap S_{\gamma_{\left(\left[g\left(x_{0}\right)\right], \nu\right)}}}\left|\left[g\left(x_{0}\right)\right]\right| d \mathcal{H}^{N-1}(y) \\
& \leqslant \int_{Q_{\nu} \cap S_{w_{n, k}}} \Psi_{1}\left(x_{0},\left[w_{n, k}(y)\right], \nu_{w_{n, k}}(y)\right) d \mathcal{H}^{N-1}(y) \\
& +C \int_{Q_{\nu}\left(0, r_{n, k}\right) \cap S_{\rho_{n, k}}}\left|\left[\rho_{n, k}(y)\right]\right| d \mathcal{H}^{N-1}(y)+C \int_{\partial Q\left(0, r_{n, k}\right)}\left|v_{n, k}(y)\right| d \mathcal{H}^{N-1}(y) \\
& +C \int_{\partial Q\left(0, r_{n, k}\right)}\left|\rho_{n, k}(y)\right| d \mathcal{H}^{N-1}(y) \\
& +C \int_{\left[Q_{\nu} \backslash Q_{\nu}\left(0, r_{n, k}\right)\right] \cap S_{\gamma_{\left(\left[g\left(x_{0}\right)\right], \nu\right)}}}\left|\left[g\left(x_{0}\right)\right]\right| d \mathcal{H}^{N-1}(y) .
\end{aligned}
$$


SECOND-ORDER STRUCTURED DEFORMATIONS: RELAXATION, INTEGRAL REPRESENTATION AND APPLICATION\&3

Since the last four integrals in the above expression converge to zero as $k, n \rightarrow+\infty$ we conclude from (5.8) that

$$
\begin{aligned}
\frac{d \mu}{d \mathcal{H}^{N-1}\left\lfloor S_{g}\right.}\left(x_{0}\right) & \geqslant \liminf _{k, n} \int_{Q_{\nu} \cap S_{\tilde{z}_{n, k}}} \Psi_{1}\left(x_{0},\left[\tilde{z}_{n, k}(y)\right], \nu_{\tilde{z}_{n, k}}(y)\right) d \mathcal{H}^{N-1}(y)+O(\varepsilon) \\
& \geqslant \gamma_{1}\left(x_{0},\left[g\left(x_{0}\right)\right], \nu\right)+O(\varepsilon)
\end{aligned}
$$

so to conclude the result it suffices to let $\varepsilon \rightarrow 0^{+}$.

5.1.2. The upper bound inequality. We now prove the upper bound inequalities for both the bulk and interfacial terms.

Proposition 5.5. For $\mathcal{L}^{N}$ a.e. $x_{0} \in \Omega$ we have that

$$
\frac{d I_{1}(g, G, \Gamma)}{d \mathcal{L}^{N}}\left(x_{0}\right) \leqslant W_{1}\left(x_{0}, G\left(x_{0}\right)-\nabla g\left(x_{0}\right)\right)
$$

Proof. Let $x_{0}$ be a point of approximate continuity for $G$ and $\nabla g$, that is,

$$
\lim _{\delta \rightarrow 0^{+}} \frac{1}{\delta^{N}} \int_{Q\left(x_{0}, \delta\right)}\left|G(x)-G\left(x_{0}\right)\right|+\left|\nabla g(x)-\nabla g\left(x_{0}\right)\right| d x=0 .
$$

Given $\varepsilon>0$ let $u \in S B V^{2}\left(\Omega ; \mathbb{R}^{d}\right)$ be such that $\left.u\right|_{\partial Q}=0, \nabla u(x)=G\left(x_{0}\right)-\nabla g\left(x_{0}\right)$ for a.e. $x \in Q$ and

$$
\int_{Q \cap S_{u}} \Psi_{1}\left(x_{0},[u(y)], \nu_{u}(y)\right) d y \leqslant W_{1}\left(x_{0}, G\left(x_{0}\right)-\nabla g\left(x_{0}\right)\right)+\varepsilon .
$$

Extend $u$ by periodicity to all of $\mathbb{R}^{N}$ and for $n \in \mathbb{N}$ and $\delta>0$ define

$$
u_{n, \delta}(x):=\frac{\delta}{n} u\left(\frac{n\left(x-x_{0}\right)}{\delta}\right) .
$$

For each $\delta>0$, by Theorem 2.4, let $v_{\delta} \in S B V\left(Q\left(x_{0}, \delta\right) ; \mathbb{R}^{d \times N}\right)$ be such that

$$
\nabla v_{\delta}=\Gamma(x)-\nabla G(x),
$$

for $\mathcal{L}^{N}$ a.e. $x \in Q\left(x_{0}, \delta\right)$, and

$$
\left\|D v_{\delta}\right\|\left(Q\left(x_{0}, \delta\right)\right) \leqslant C(N) \int_{Q\left(x_{0}, \delta\right)}|\Gamma(x)-\nabla G(x)| d x .
$$

By Lemma 2.3 let $v_{k, \delta}: Q\left(x_{0}, \delta\right) \rightarrow \mathbb{R}^{d \times N}$ be a sequence of piecewise constant functions such that

$$
v_{k, \delta} \underset{k \rightarrow+\infty}{\stackrel{L^{1}}{\rightarrow}}-v_{\delta}
$$

and

$$
\lim _{k \rightarrow+\infty}\left\|D v_{k, \delta}\right\|\left(Q\left(x_{0}, \delta\right)\right)=\left\|D v_{\delta}\right\|\left(Q\left(x_{0}, \delta\right)\right) .
$$

Applying once more Theorem 2.4 let $\rho_{k, \delta} \in S B V^{2}\left(Q\left(x_{0}, \delta\right) ; \mathbb{R}^{d}\right)$ be such that

$$
\nabla \rho_{k, \delta}(x)=G(x)-G\left(x_{0}\right)+\nabla g\left(x_{0}\right)-\nabla g(x)+v_{\delta}(x)+v_{k, \delta}(x),
$$

for $\mathcal{L}^{N}$ a.e. $x \in Q\left(x_{0}, \delta\right)$, and

$$
\left\|D \rho_{k, \delta}\right\|\left(Q\left(x_{0}, \delta\right)\right) \leqslant C(N) \int_{Q\left(x_{0}, \delta\right)}\left|G(x)-G\left(x_{0}\right)\right|+\left|\nabla g(x)-\nabla g\left(x_{0}\right)\right|+\left|v_{\delta}(x)+v_{k, \delta}(x)\right| d x .
$$

By (5.13), for each $\delta>0$ we can choose $k=k(\delta)$ large enough so that

$$
\int_{Q\left(x_{0}, \delta\right)}\left|v_{\delta}(x)+v_{k, \delta}(x)\right| d x \leqslant \delta^{N+1} .
$$

Thus, defining $\rho_{\delta}:=\rho_{\delta, k(\delta)}$, by (5.10) and (5.15) it follows that

$$
\lim _{\delta \rightarrow 0^{+}} \frac{\left\|D \rho_{\delta}\right\|\left(Q\left(x_{0}, \delta\right)\right)}{\delta^{N}}=0 .
$$


Again by Lemma 2.3, let $\rho_{n, \delta}$ be a sequence of piecewise constant functions such that, for all $\delta>0$,

$$
\rho_{n, \delta} \underset{n \rightarrow+\infty}{\stackrel{L^{1}}{\longrightarrow}}-\rho_{\delta} \quad \text { and }\left\|D \rho_{n, \delta}\right\|\left(Q\left(x_{0}, \delta\right)\right) \underset{n \rightarrow+\infty}{\longrightarrow}\left\|D \rho_{\delta}\right\|\left(Q\left(x_{0}, \delta\right)\right) .
$$

Now define, for $x \in Q\left(x_{0}, \delta\right)$,

$$
w_{n, \delta}(x):=g(x)+u_{n, \delta}(x)+\rho_{\delta}(x)+\rho_{n, \delta}(x) .
$$

By periodicity, $w_{n, \delta} \underset{n \rightarrow+\infty}{\stackrel{L^{1}}{\longrightarrow}} g$ since,

$$
\int_{Q\left(x_{0}, \delta\right)}\left|u_{n, \delta}(x)\right| d x=\frac{\delta^{N+1}}{n} \int_{Q}|u(y)| d y \underset{n \rightarrow+\infty}{\longrightarrow} 0 .
$$

Notice also that $\nabla^{2} w_{n, \delta}=\Gamma$, and it is easy to verify that $\nabla w_{n, \delta} \underset{n \rightarrow+\infty}{\longrightarrow} G$ in $L^{1}\left(Q\left(x_{0}, \delta\right) ; \mathbb{R}^{d}\right)$.

Thus the sequence $w_{n, \delta}$ is admissible for $I_{1}\left(g, G, \Gamma, Q\left(x_{0}, \delta\right)\right)$ and so, by (H8), we have

$$
\begin{aligned}
\frac{d I_{1}(g, G, \Gamma)}{d \mathcal{L}^{N}}\left(x_{0}\right) & =\lim _{\delta \rightarrow 0^{+}} \frac{I_{1}\left(g, G, \Gamma, Q\left(x_{0}, \delta\right)\right)}{\delta^{N}} \\
& \leqslant \lim _{\delta \rightarrow 0^{+}} \liminf _{n \rightarrow+\infty}\left[\frac{1}{\delta^{N}} \int_{S_{w_{n}, \delta} \cap Q\left(x_{0}, \delta\right)} \Psi_{1}\left(x,\left[w_{n, \delta}(x)\right], \nu_{w_{n, \delta}}(x)\right) d \mathcal{H}^{N-1}(x)\right] \\
& \leqslant \liminf _{\delta \rightarrow 0^{+}} \liminf _{n \rightarrow+\infty}\left[\frac{1}{\delta^{N}} \int_{S_{g} \cap Q\left(x_{0}, \delta\right)} \Psi_{1}\left(x,[g(x)], \nu_{g}(x)\right) d \mathcal{H}^{N-1}(x)\right. \\
& +\frac{1}{\delta^{N}} \int_{\left\{x_{0}+\frac{\delta}{n} S_{u}\right\} \cap Q\left(x_{0}, \delta\right)} \Psi_{1}\left(x, \frac{\delta}{n}\left[u\left(\frac{n\left(x-x_{0}\right)}{\delta}\right)\right], \nu_{u}\left(\frac{n\left(x-x_{0}\right)}{\delta}\right)\right) d \mathcal{H}^{N-1}(x) \\
& +\frac{1}{\delta^{N}} \int_{S_{\rho_{\delta}} \cap Q\left(x_{0}, \delta\right)} \Psi_{1}\left(x,\left[\rho_{\delta}(x)\right], \nu_{\rho_{\delta}}(x)\right) d \mathcal{H}^{N-1}(x) \\
& \left.+\frac{1}{\delta^{N}} \int_{S_{\rho_{n, \delta}} \cap Q\left(x_{0}, \delta\right)} \Psi_{1}\left(x,\left[\rho_{n, \delta}(x)\right], \nu_{\rho_{n, \delta}}(x)\right) d \mathcal{H}^{N-1}(x)\right]
\end{aligned}
$$

Since $\frac{d\left\|D^{s} g\right\|}{d \mathcal{L}^{N}}\left(x_{0}\right)=0$, by (H5) we conclude that

$$
\begin{aligned}
\frac{1}{\delta^{N}} \int_{S_{g} \cap Q\left(x_{0}, \delta\right)} \Psi_{1}\left(x,[g(x)], \nu_{g}(x)\right) d \mathcal{H}^{N-1}(x) & \leqslant \frac{1}{\delta^{N}} \int_{S_{g} \cap Q\left(x_{0}, \delta\right)} C|[g(x)]| d \mathcal{H}^{N-1}(x) \\
& \leqslant C \frac{\left\|D^{s} g\right\|\left(Q\left(x_{0}, \delta\right)\right)}{\mathcal{L}^{N}\left(Q\left(x_{0}, \delta\right)\right)} \underset{\delta \rightarrow 0^{+}}{\longrightarrow} 0 .
\end{aligned}
$$

Moreover, once again hypothesis (H5), together with (5.16) and (5.17), also yields

$$
\lim _{\delta \rightarrow 0^{+}} \frac{1}{\delta^{N}} \int_{S_{\rho_{\delta}} \cap Q\left(x_{0}, \delta\right)} \Psi_{1}\left(x,\left[\rho_{\delta}(x)\right], \nu_{\rho_{\delta}}(x)\right) d \mathcal{H}^{N-1}(x)=0,
$$

and

$$
\lim _{\delta \rightarrow 0^{+}} \lim _{n \rightarrow+\infty} \frac{1}{\delta^{N}} \int_{S_{\rho_{n, \delta}} \cap Q\left(x_{0}, \delta\right)} \Psi_{1}\left(x,\left[\rho_{n, \delta}(x)\right], \nu_{\rho_{n, \delta}}(x)\right) d \mathcal{H}^{N-1}(x)=0 .
$$

Finally, changing variables, using the periodicity of $u$, (H7) and (5.11), we obtain

$$
\begin{aligned}
& \frac{1}{\delta^{N}} \int_{\left\{x_{0}+\frac{\delta}{n} S_{u}\right\} \cap Q\left(x_{0}, \delta\right)} \Psi_{1}\left(x, \frac{\delta}{n}\left[u\left(\frac{n\left(x-x_{0}\right)}{\delta}\right)\right], \nu_{u}\left(\frac{n\left(x-x_{0}\right)}{\delta}\right)\right) d \mathcal{H}^{N-1}(x) \\
& =\frac{1}{n^{N}} \int_{n Q \cap S_{u}} \Psi_{1}\left(x_{0}+\frac{\delta}{n} y,[u(y)], \nu_{u}(y)\right) d \mathcal{H}^{N-1}(y)
\end{aligned}
$$


SECOND-ORDER STRUCTURED DEFORMATIONS: RELAXATION, INTEGRAL REPRESENTATION AND APPLICATION\&5

$$
\begin{aligned}
& =\int_{Q \cap S_{u}} \Psi_{1}\left(x_{0},[u(y)], \nu_{u}(y)\right) d \mathcal{H}^{N-1}(y) \\
& \quad+\int_{Q \cap S_{u}} \Psi_{1}\left(x_{0}+\frac{\delta}{n} y,[u(y)], \nu_{u}(y)\right)-\Psi_{1}\left(x_{0},[u(y)], \nu_{u}(y)\right) d \mathcal{H}^{N-1}(y) \\
& \leqslant W_{1}\left(x_{0}, G\left(x_{0}\right)-\nabla g\left(x_{0}\right)\right)+\varepsilon \\
& \quad+\int_{Q \cap S_{u}} \Psi_{1}\left(x_{0}+\frac{\delta}{n} y,[u(y)], \nu_{u}(y)\right)-\Psi_{1}\left(x_{0},[u(y)], \nu_{u}(y)\right) d \mathcal{H}^{N-1}(y),
\end{aligned}
$$

where, by (H6) and for $\delta$ small enough,

$$
\begin{aligned}
& \left|\int_{Q \cap S_{u}} \Psi_{1}\left(x_{0}+\frac{\delta}{n} y,[u(y)], \nu_{u}(y)\right)-\Psi_{1}\left(x_{0},[u(y)], \nu_{u}(y)\right) d \mathcal{H}^{N-1}(y)\right| \\
& \leqslant \varepsilon C \int_{Q \cap S_{u}}|[u(y)]| d \mathcal{H}^{N-1}(y) \leqslant \varepsilon C\|D u\|(Q) .
\end{aligned}
$$

Thus the result follows by letting $\varepsilon \rightarrow 0^{+}$.

Proposition 5.6. For $\mathcal{H}^{N-1}$ a.e. $x_{0} \in S_{g}$ we have that

$$
\frac{d I_{1}(g, G, \Gamma)}{d \mathcal{H}^{N-1}\left\lfloor S_{g}\right.}\left(x_{0}\right) \leqslant \gamma_{1}\left(x_{0},\left[g\left(x_{0}\right)\right], \nu_{g}\left(x_{0}\right)\right) .
$$

Proof. Following an argument of Ambrosio, Mortola and Tortorelli [5], it suffices to prove (5.18) when $g=\lambda \chi_{E}$ where $\lambda \in \mathbb{R}^{d}$ and $\chi_{E}$ is the characteristic function of a set of finite perimeter $E$. We start by addressing the case where $E$ is a polyhedral set. Let $x_{0} \in S_{g}$ be such that

$$
\lim _{\delta \rightarrow 0^{+}} \frac{1}{\delta^{N-1}} \int_{Q_{\nu}\left(x_{0}, \delta\right)}|G(x)| d x=0
$$

where we are denoting by $\nu:=\nu_{g}\left(x_{0}\right)$, and $\left[g\left(x_{0}\right)\right]=\lambda$. By definition of $\gamma_{1}\left(x_{0}, \lambda, \nu\right)$, given $\varepsilon>0$, consider $u \in S B V^{2}\left(Q_{\nu} ; \mathbb{R}^{d}\right)$ such that $u_{\mid \partial Q_{\nu}}(x)=\gamma_{(\lambda, \nu)}(x), \nabla u=0$ a.e. in $Q_{\nu}$, and

$$
\int_{Q_{\nu}} \Psi_{1}\left(x_{0},[u(y)], \nu_{u}(y)\right) d \mathcal{H}^{N-1}(y) \leqslant \gamma_{1}\left(x_{0}, \lambda, \nu\right)+\varepsilon
$$

For $\delta>0$ small enough, and $n \in \mathbb{N}$, define

and let

$$
\begin{aligned}
& D_{\nu}^{n}\left(x_{0}, \delta\right):=Q_{\nu}\left(x_{0}, \delta\right) \cap\left\{x: \frac{\left|\left(x-x_{0}\right) \cdot \nu\right|}{\delta}<\frac{1}{2 n}\right\}, \\
& Q_{\nu}^{+}\left(x_{0}, \delta\right):=Q_{\nu}\left(x_{0}, \delta\right) \cap\left\{x: \frac{\left(x-x_{0}\right) \cdot \nu}{\delta}>0\right\}, \\
& Q_{\nu}^{-}\left(x_{0}, \delta\right):=Q_{\nu}\left(x_{0}, \delta\right) \cap\left\{x: \frac{\left(x-x_{0}\right) \cdot \nu}{\delta}<0\right\},
\end{aligned}
$$

$$
u_{n, \delta}(x):= \begin{cases}\lambda & x \in Q_{\nu}^{+}\left(x_{0}, \delta\right) \backslash D_{\nu}^{n}\left(x_{0}, \delta\right), \\ u\left(\frac{n\left(x-x_{0}\right)}{\delta}\right) & x \in D_{\nu}^{n}\left(x_{0}, \delta\right), \\ 0 & x \in Q_{\nu}^{-}\left(x_{0}, \delta\right) \backslash D_{\nu}^{n}\left(x_{0}, \delta\right),\end{cases}
$$

where $u$ has been extended by $Q$-periodicity to all of $\mathbb{R}^{N}$. Notice that, by periodicity of $u$,

$$
\lim _{n \rightarrow+\infty}\left\|u_{n, \delta}-\tilde{\gamma}_{(\lambda, \nu)}\right\|_{L^{1}\left(Q_{\nu}\left(x_{0}, \delta\right) ; \mathbb{R}^{d}\right)}=0,
$$

where $\tilde{\gamma}_{(\lambda, \nu)}(x):=\gamma_{(\lambda, \nu)}\left(x-x_{0}\right)$.

By Theorem 2.4, let $v_{\delta} \in S B V\left(Q_{\nu}\left(x_{0}, \delta\right) ; \mathbb{R}^{d \times N}\right)$ be such that

$$
\nabla v_{\delta}=\Gamma(x)-\nabla G(x),
$$

for $\mathcal{L}^{N}$ a.e. $x \in Q_{\nu}\left(x_{0}, \delta\right)$, and

$$
\left\|D v_{\delta}\right\|\left(Q\left(x_{0}, \delta\right)\right) \leqslant C(N) \int_{Q_{\nu}\left(x_{0}, \delta\right)}|\Gamma(x)-\nabla G(x)| d x .
$$


By Lemma 2.3, let $v_{n, \delta} \in S B V\left(Q\left(x_{0}, \delta\right) ; \mathbb{R}^{d \times N}\right)$ be a sequence of piecewise constant functions such that

$$
v_{n, \delta} \underset{n \rightarrow+\infty}{\stackrel{L^{1}}{\longrightarrow}}-v_{\delta},
$$

and

$$
\lim _{n \rightarrow+\infty}\left\|D v_{n, \delta}\right\|\left(Q_{\nu}\left(x_{0}, \delta\right)\right)=\left\|D v_{\delta}\right\|\left(Q\left(x_{0}, \delta\right)\right) .
$$

Applying again Theorem 2.4, let $\rho_{n, \delta} \in S B V^{2}\left(Q_{\nu}\left(x_{0}, \delta\right) ; \mathbb{R}^{d}\right)$ be such that

$$
\nabla \rho_{n, \delta}(x)=G(x)+v_{\delta}(x)+v_{n, \delta}(x),
$$

for $\mathcal{L}^{N}$ a.e. $x \in Q_{\nu}\left(x_{0}, \delta\right)$, and

$$
\left\|D \rho_{n, \delta}\right\|\left(Q_{\nu}\left(x_{0}, \delta\right)\right) \leqslant C(N) \int_{Q_{\nu}\left(x_{0}, \delta\right)}|G(x)|+\left|v_{\delta}(x)+v_{n, \delta}(x)\right| d x .
$$

Notice that $\nabla^{2} \rho_{n, \delta}(x)=\Gamma(x)$. By (5.23), for each $\delta$ we can choose $n(\delta)$ such that

$$
\int_{Q_{\nu}\left(x_{0}, \delta\right)}\left|v_{\delta}(x)+v_{n(\delta), \delta}(x)\right| d x \leqslant \delta^{N} .
$$

Then, writing for simplicity $\rho_{\delta}$ instead of $\rho_{n(\delta), \delta}$, by (5.25) and (5.19) we have that

$$
\lim _{\delta \rightarrow 0^{+}} \frac{\left\|D \rho_{\delta}\right\|\left(Q_{\nu}\left(x_{0}, \delta\right)\right)}{\delta^{N-1}}=0 .
$$

By Lemma 2.3, let $\tilde{\rho}_{n, \delta} \in S B V\left(Q_{\nu}\left(x_{0}, \delta\right) ; \mathbb{R}^{d}\right)$ be a sequence of piecewise constant functions such that, for all $\delta>0$,

$$
\tilde{\rho}_{n, \delta} \underset{n \rightarrow+\infty}{\stackrel{L^{1}}{\longrightarrow}}-\rho_{\delta} \text { and } \lim _{n \rightarrow+\infty}\left\|D \tilde{\rho}_{n, \delta}\right\|\left(Q_{\nu}\left(x_{0}, \delta\right)\right)=\left\|D \rho_{\delta}\right\|\left(Q_{\nu}\left(x_{0}, \delta\right)\right) .
$$

Now, for $x \in Q_{\nu}\left(x_{0}, \delta\right)$, define the sequence

$$
w_{n, \delta}(x):=u_{n, \delta}(x)+\rho_{\delta}(x)+\tilde{\rho}_{n, \delta}(x) .
$$

We point out that

$$
\lim _{n \rightarrow+\infty}\left\|w_{n, \delta}-\tilde{\gamma}_{(\lambda, \nu)}\right\|_{L^{1}\left(Q_{\nu}\left(x_{0}, \delta\right) ; \mathbb{R}^{d}\right)}=\lim _{n \rightarrow+\infty}\left\|w_{n, \delta}-g\right\|_{L^{1}\left(Q_{\nu}\left(x_{0}, \delta\right) ; \mathbb{R}^{d}\right)}=0,
$$

that

$$
\lim _{n \rightarrow+\infty}\left\|\nabla w_{n, \delta}-G\right\|_{L^{1}\left(Q_{\nu}\left(x_{0}, \delta\right) ; \mathbb{R}^{d \times N}\right)}=0,
$$

and that $\nabla^{2} w_{n, \delta}=\Gamma$, hence the sequence $w_{n, \delta}$ is admissible for $I_{1}\left(g, G, \Gamma, Q_{\nu}\left(x_{0}, \delta\right)\right)$. Therefore we have, by (H8) and (H5),

$$
\begin{aligned}
& \frac{d I_{1}(g, G, \Gamma)}{d \mathcal{H}^{N-1}\left\lfloor S_{g}\right.}\left(x_{0}\right)=\lim _{\delta \rightarrow 0^{+}} \frac{I_{1}\left(g, G, \Gamma, Q_{\nu}\left(x_{0}, \delta\right)\right)}{\delta^{N-1}} \\
& \leqslant \lim _{\delta \rightarrow 0^{+}} \liminf _{n \rightarrow+\infty} \frac{1}{\delta^{N-1}} \int_{S_{w_{n, \delta}} \cap Q_{\nu}\left(x_{0}, \delta\right)} \Psi_{1}\left(x,\left[w_{n, \delta}(x)\right], \nu_{w_{n, \delta}}(x)\right) d \mathcal{H}^{N-1}(x) \\
& \leqslant \limsup _{\delta \rightarrow 0^{+}} \limsup _{n \rightarrow+\infty} \frac{1}{\delta^{N-1}} \int_{S_{u_{n, \delta} \cap Q_{\nu}\left(x_{0}, \delta\right)}} \Psi_{1}\left(x,\left[u_{n, \delta}(x)\right], \nu_{\left(u_{n, \delta}\right)}(x)\right) d \mathcal{H}^{N-1}(x) \\
& +\limsup _{\delta \rightarrow 0^{+}} \limsup _{n \rightarrow+\infty} \frac{1}{\delta^{N-1}} \int_{S_{\rho_{\delta}+\tilde{\rho}_{n, \delta}} \cap Q_{\nu}\left(x_{0}, \delta\right)} \Psi_{1}\left(x,\left[\rho_{\delta}(x)+\tilde{\rho}_{n, \delta}(x)\right], \nu_{\rho_{\delta}+\tilde{\rho}_{n, \delta}}(x)\right) d \mathcal{H}^{N-1}(x) \\
& \leqslant \limsup _{\delta \rightarrow 0^{+}} \limsup _{n \rightarrow+\infty} \frac{1}{\delta^{N-1}} \int_{\left\{x: \frac{n\left(x-x_{0}\right)}{\delta} \in S_{u}\right\} \cap D_{\nu}^{n}\left(x_{0}, \delta\right)} \Psi_{1}\left(x,\left[u\left(\frac{n\left(x-x_{0}\right)}{\delta}\right)\right], \nu_{u}\left(\frac{n\left(x-x_{0}\right)}{\delta}\right)\right) d \mathcal{H}^{N-1}(x) \\
& +\limsup _{\delta \rightarrow 0^{+}} \limsup _{n \rightarrow+\infty} \frac{1}{\delta^{N-1}} \int_{S_{\rho_{\delta}+\tilde{\rho}_{n, \delta}} \cap Q\left(x_{0}, \delta\right)}^{C\left|\left[\rho_{\delta}(x)+\tilde{\rho}_{n, \delta}(x)\right]\right| d \mathcal{H}^{N-1}(x) .}
\end{aligned}
$$


By (5.26) and (5.27) the integral in the last line vanishes in the limit, while by changing variables setting $y:=\frac{n\left(x-x_{0}\right)}{\delta}$, we obtain by (H6), for $\delta$ small enough,

$$
\begin{aligned}
& \limsup _{\delta \rightarrow 0^{+}} \limsup _{n \rightarrow+\infty} \frac{1}{\delta^{N-1}} \int_{\left\{x: \frac{n\left(x-x_{0}\right)}{\delta} \in S_{u}\right\} \cap D_{\nu}^{n}\left(x_{0}, \delta\right)} \Psi_{1}\left(x,\left[u\left(\frac{n\left(x-x_{0}\right)}{\delta}\right)\right], \nu_{u}\left(\frac{n\left(x-x_{0}\right)}{\delta}\right)\right) d \mathcal{H}^{N-1}(x) \\
& \leqslant \limsup _{\delta \rightarrow 0^{+}} \limsup _{n \rightarrow+\infty} \frac{1}{n^{N-1}} \int_{S_{u} \cap\left\{y \in n Q_{\nu}:|y \cdot \nu| \leqslant \frac{1}{2}\right\}} \Psi_{1}\left(x_{0}+\frac{\delta}{n} y,[u(y)], \nu_{u}(y)\right) d \mathcal{H}^{N-1}(y) \\
& \leqslant \limsup _{\delta \rightarrow 0^{+}} \limsup _{n \rightarrow+\infty} \frac{1}{n^{N-1}}\left[\int_{S_{u} \cap\left\{y \in n Q_{\nu}:|y \cdot \nu| \leqslant \frac{1}{2}\right\}} \Psi_{1}\left(x_{0},[u(y)], \nu_{u}(y)\right) d \mathcal{H}^{N-1}(y)\right. \\
& \left.\quad+\int_{S_{u} \cap\left\{y \in n Q_{\nu}:|y \cdot \nu| \leqslant \frac{1}{2}\right\}} \varepsilon C|[u(y)]| d \mathcal{H}^{N-1}(y)\right] \\
& =\int_{S_{u} \cap Q_{\nu}} \Psi_{1}\left(x_{0},[u(y)], \nu_{u}(y)\right) d \mathcal{H}^{N-1}(y)+\int_{S_{u} \cap Q_{\nu}} \varepsilon C|[u(y)]| d \mathcal{H}^{N-1}(y) \\
& \leqslant \gamma_{1}\left(x_{0}, \lambda, \nu\right)+O(\varepsilon),
\end{aligned}
$$

where we have used the periodicity of $u$ and $(5.20$. The conclusion follows by the arbitrariness of $\varepsilon$.

We now assume that $g=\lambda \chi_{E}$ where $E$ is an arbitrary set of finite perimeter. Let $x_{0} \in S_{g}$ be such that

$$
\lim _{\delta \rightarrow 0^{+}} \frac{1}{\delta^{N-1}} \int_{Q_{\nu}\left(x_{0}, \delta\right)}|G(x)| d x=0
$$

where we are denoting by $\nu:=\nu_{g}\left(x_{0}\right)$. By Theorem 2.2, let $E_{n}$ be a sequence of polyhedral sets such that $\lim _{n \rightarrow+\infty} \operatorname{Per}_{\Omega}\left(E_{n}\right)=\operatorname{Per}_{\Omega}(E), \mathcal{L}^{N}\left(E_{n}\right)=\mathcal{L}^{N}(E)$ and $\chi_{E_{n}} \rightarrow \chi_{E}$ in $L^{1}(\Omega)$, as $n \rightarrow+\infty$. Let $g_{n}=\lambda \chi_{E_{n}}$, then $\lim _{n \rightarrow+\infty} g_{n}=g$ in $L^{1}\left(\Omega ; \mathbb{R}^{d}\right)$. Hence, given $U \in \mathcal{O}(\Omega)$ by Propositions 4.7 and 4.8 , we have

$$
\begin{aligned}
I_{1}(g, G, \Gamma, U) & \leqslant \liminf _{n \rightarrow+\infty} I_{1}\left(g_{n}, G, \Gamma, U\right) \\
& \leqslant \liminf _{n \rightarrow+\infty}\left[\int_{U} W_{1}(x, G(x)) d x+\int_{U \cap S_{g_{n}}} \gamma_{1}\left(x,\left[g_{n}(x)\right], \nu_{g_{n}}(x)\right) d \mathcal{H}^{N-1}(x)\right] \\
& \leqslant C \int_{U}|G(x)| d x+\limsup _{n \rightarrow+\infty} \int_{U \cap S_{g_{n}}} \gamma_{1}\left(x,\left[g_{n}(x)\right], \nu_{g_{n}}(x)\right) d \mathcal{H}^{N-1}(x) .
\end{aligned}
$$

Recall that by Remark 4.10 there exists a non-increasing sequence of continuous functions $\gamma_{1}^{m}: \Omega \times \mathbb{R}^{N} \rightarrow[0,+\infty)$ such that

$$
\gamma_{1}(x, \lambda, \theta)=\inf _{m} \gamma_{1}^{m}(x, \theta)=\lim _{m} \gamma_{1}^{m}(x, \theta) \leqslant C|\theta|, \forall(x, \theta) \in \Omega \times \mathbb{R}^{N} .
$$

Thus, by Theorem 2.1, it follows from $(5.29$ that

$$
\begin{aligned}
I_{1}(g, G, \Gamma, U) & \leqslant C \int_{U}|G(x)| d x+\limsup _{n \rightarrow+\infty} \int_{U \cap S_{g_{n}}} \gamma_{1}^{m}\left(x, \nu_{g_{n}}(x)\right) d \mathcal{H}^{N-1}(x) \\
& \leqslant C \int_{U}|G(x)| d x+\int_{U \cap S_{g}} \gamma_{1}^{m}\left(x, \nu_{g}(x)\right) d \mathcal{H}^{N-1}(x) .
\end{aligned}
$$

Letting $m \rightarrow+\infty$ and using the monotone convergence theorem we conclude that

$$
I_{1}(g, G, \Gamma, U) \leqslant C \int_{U}|G(x)| d x+\int_{U \cap S_{g}} \gamma_{1}\left(x, \nu_{g}(x)\right) d \mathcal{H}^{N-1}(x) .
$$


Using (5.28) and Proposition 4.9 we finally obtain

$$
\begin{aligned}
\frac{d I_{1}(g, G, \Gamma)}{d \mathcal{H}^{N-1}\left\lfloor S_{g}\right.}\left(x_{0}\right) & =\lim _{\delta \rightarrow 0^{+}} \frac{1}{\delta^{N-1}} I_{1}\left(g, G, \Gamma, Q_{\nu}\left(x_{0}, \delta\right)\right) \\
& \leqslant \lim _{\delta \rightarrow 0^{+}} \frac{1}{\delta^{N-1}} \int_{Q_{\nu}\left(x_{0}, \delta\right) \cap S_{g}} \gamma_{1}\left(x, \nu_{g}(x)\right) d \mathcal{H}^{N-1}(x) \\
& \leqslant \gamma_{1}\left(x_{0},\left[g\left(x_{0}\right)\right], \nu_{g}\left(x_{0}\right)\right)+O(\varepsilon),
\end{aligned}
$$

and the result follows by letting $\varepsilon \rightarrow 0^{+}$.

\subsection{Integral representation of $I_{2}$ •}

Theorem 5.7. Under hypotheses (H1)-(H8) we have

$$
I_{2}(G, \Gamma)=\int_{\Omega} W_{2}(x, G(x), \nabla G(x), \Gamma(x)) d x+\int_{S_{G} \cap \Omega} \gamma_{2}\left(x, G(x),[G(x)], \nu_{G}(x)\right) d \mathcal{H}^{N-1}(x) .
$$

Proof. The proof of the above integral representation for $I_{2}$ is similar to that of $I_{1}$ so we will only outline the proof.

In order to obtain a lower bound for the bulk term we start by fixing a point $x_{0}$, which is chosen to be a point of approximate differentiability of $G$ and of approximate continuity of $\Gamma$. Starting from a sequence $v_{n}$ for which

$$
\lim _{n \rightarrow+\infty}\left[\int_{\Omega} W\left(x, G(x), \nabla v_{n}(x)\right) d x+\int_{S_{v_{n}} \cap \Omega} \Psi_{2}\left(x,\left[v_{n}(x)\right], \nu_{v_{n}}(x)\right) d \mathcal{H}^{N-1}(x)\right]<+\infty
$$

we construct a new sequence $u_{n, k}$ so that

$$
\begin{aligned}
& \frac{d I_{2}(G, \Gamma)}{d \mathcal{L}^{N}}\left(x_{0}\right) \\
& \geqslant \lim _{k, n}\left[\int_{Q} W\left(x_{0}, G\left(x_{0}\right), \nabla u_{n, k}(x)\right) d x+\int_{S_{u_{n, k}} \cap Q} \Psi_{2}\left(x_{0},\left[u_{n, k}(x)\right], \nu_{u_{n, k}}(x)\right) d \mathcal{H}^{N-1}(x)\right]+O(\varepsilon),
\end{aligned}
$$

where we use hypotheses (H2) and (H6) to fix $x_{0}$ and $G\left(x_{0}\right)$. We further modify $u_{n, k}$ in order to obtain a sequence $z_{n, k}$ which is admissible for $W_{2}\left(x_{0}, G\left(x_{0}\right), \nabla G\left(x_{0}\right), \Gamma\left(x_{0}\right)\right)$. This is achieved by setting $z_{n, k}(x)$ equal to $\nabla G\left(x_{0}\right) \cdot x$ near the boundary of $Q$ and equal to $u_{n, k}(x)+C_{n, k} \cdot x$ in a smaller cube of the form $Q\left(0, r_{n, k}\right)$, where $C_{n, k}$ is chosen so that

$$
\int_{Q} \nabla z_{n, k}(x) d x=\Gamma\left(x_{0}\right) .
$$

Hypotheses (H2) and (H5) and a careful selection of the side-length of the smaller cube $r_{n, k}$ guarantee that the energy does not increase when $u_{n, k}$ is replaced by $z_{n, k}$ so the result follows by letting $\varepsilon \rightarrow 0^{+}$.

Regarding the lower bound for the interfacial term we fix a point $x_{0}$, which is chosen to be a point of approximate continuity of $G$, and such that

$$
\lim _{\delta \rightarrow 0^{+}} \frac{\mathcal{H}^{N-1}\left(S_{G} \cap Q_{\nu}\left(x_{0}, \delta\right)\right)}{\delta^{N-1}}=1
$$

and

$$
\lim _{\delta \rightarrow 0^{+}} \frac{1}{\delta^{N-1}} \int_{Q_{\nu}\left(x_{0}, \delta\right)}|\Gamma(x)| d x=0,
$$

where $\nu:=\nu_{G}\left(x_{0}\right)$. Starting from the sequence $v_{n}$ in (5.30), the properties of $x_{0}$, together with hypotheses (H2) and (H6), yield a new sequence $w_{n, k}$ satisfying

$$
\begin{aligned}
& \frac{d I_{2}(G, \Gamma)}{d \mathcal{H}^{N-1}\left[S_{G}\right.}\left(x_{0}\right) \\
& \geqslant \lim _{k, n}\left[\int_{Q_{\nu}} W^{\infty}\left(x_{0}, G\left(x_{0}\right), \nabla w_{n, k}(x)\right) d x+\int_{S_{w_{n, k}} \cap Q_{\nu}} \Psi_{2}\left(x_{0},\left[w_{n, k}(x)\right], \nu_{w_{n, k}}(x)\right) d \mathcal{H}^{N-1}(x)\right]+O(\varepsilon),
\end{aligned}
$$


in this step hypothesis (H4) comes into play. As above, $w_{n, k}$ is further modified in order to obtain a sequence $z_{n, k}$ which is admissible for $\gamma_{2}\left(x_{0}, G\left(x_{0}\right),\left[G\left(x_{0}\right)\right], \nu_{G}\left(x_{0}\right)\right)$. This is achieved by setting $z_{n, k}(x)$ equal to $\gamma_{\left(\left[G\left(x_{0}\right)\right], \nu\right)}$ near the boundary of $Q_{\nu}$ and equal to $w_{n, k}(x)+C_{n, k} \cdot x$ in a smaller cube of the form $Q\left(0, r_{n, k}\right)$, where $C_{n, k}$ is chosen so that

$$
\int_{Q_{\nu}} \nabla z_{n, k}(x) d x=0
$$

Due to hypotheses (H2) and (H5), the replacement of $w_{n, k}$ by $z_{n, k}$ does not translate into an increase in energy, so the result follows by letting $\varepsilon \rightarrow 0^{+}$.

For the upper bound for the bulk term we fix a point $x_{0}$ of approximate continuity of both $G$ and $\Gamma$ and, for $\varepsilon>0$, we let $v \in S B V\left(Q ; \mathbb{R}^{d \times N}\right)$ be such that $v(x)=\nabla G\left(x_{0}\right) \cdot x$ on $\partial Q$, $\int_{Q} \nabla v(x) d x=\Gamma\left(x_{0}\right)$ and

$$
\begin{aligned}
\int_{Q} W\left(x_{0}, G\left(x_{0}\right), \nabla v(x)\right) d x+ & \int_{S_{v} \cap Q} \Psi_{2}\left(x_{0},[v(x)], \nu_{v}(x)\right) d \mathcal{H}^{N-1}(x) \\
& \leqslant W_{2}\left(x_{0}, G\left(x_{0}\right), \nabla G\left(x_{0}\right), \Gamma\left(x_{0}\right)\right)+\varepsilon .
\end{aligned}
$$

Extending $v$ by periodicity to all of $\mathbb{R}^{N}$ and using Theorem 2.4 and Lemma 2.3 we construct a sequence $w_{n, \delta}$ so that

$$
\begin{aligned}
& \frac{d I_{2}(G, \Gamma)}{d \mathcal{L}^{N}}\left(x_{0}\right) \\
& \leqslant \limsup _{\delta \rightarrow 0^{+}} \limsup _{n \rightarrow+\infty} \frac{1}{\delta^{N}}\left[\int_{Q\left(x_{0}, \delta\right)} W\left(x, G(x), \nabla w_{n, \delta}(x)\right) d x\right. \\
& \left.+\int_{Q\left(x_{0}, \delta\right) \cap S_{w_{n, \delta}}} \Psi_{2}\left(x,\left[w_{n, \delta}(x)\right], \nu_{w_{n, \delta}}(x)\right) d \mathcal{H}^{N-1}(x)\right] \\
& \leqslant \int_{Q} W\left(x_{0}, G\left(x_{0}\right), \nabla v(x)\right) d x+\int_{S_{v} \cap Q} \Psi_{2}\left(x_{0},[v(x)], \nu_{v}(x)\right) d \mathcal{H}^{N-1}(x)+O(\varepsilon),
\end{aligned}
$$

where we use hypotheses (H2) and (H6) to fix $x_{0}$ and $G\left(x_{0}\right)$, and periodicity arguments. Hence, by (5.31) and given the arbitrariness of $\varepsilon$, we conclude the desired inequality.

As in the case of $I_{1}$, the upper bound for the interfacial term of $I_{2}$ is proved in two steps, first for $G=\Lambda \chi_{E}$, where $E$ is a polyhedral set, and then generalized to an arbitrary set of finite perimeter $E$, by using Theorem 2.2, Propositions 4.7, 4.11, 4.13, as well as Remark 4.13 and Theorem 2.1.

To prove the first step, fix a point $x_{0}$ such that

$$
\lim _{\delta \rightarrow 0^{+}} \frac{1}{\delta^{N-1}} \int_{Q_{\nu}\left(x_{0}, \delta\right)}|\Gamma(x)|+|G(x)|+|\nabla G(x)| d x=0,
$$

where $\nu:=\nu_{G}\left(x_{0}\right)$. Given $\varepsilon>0$, we let $v \in S B V\left(Q_{\nu} ; \mathbb{R}^{d \times N}\right)$ be such that $v(x)=\gamma_{(\Lambda, \nu)}$ on $\partial Q_{\nu}, \int_{Q_{\nu}} \nabla v(x) d x=0$ and

$$
\begin{aligned}
\int_{Q_{\nu}} W^{\infty}\left(x_{0}, G\left(x_{0}\right), \nabla v(x)\right) d x+ & \int_{S_{v} \cap Q_{\nu}} \Psi_{2}\left(x_{0},[v(x)], \nu_{v}(x)\right) d \mathcal{H}^{N-1}(x) \\
& \leqslant \gamma_{2}\left(x_{0}, G\left(x_{0}\right), \Lambda, \nu\right)+\varepsilon .
\end{aligned}
$$

Extending $v$ by periodicity to all of $\mathbb{R}^{N}$, and using the usual combination of Theorem 2.4 and Lemma 2.3, we construct a sequence $w_{n, \delta}$ so that

$$
\begin{aligned}
& \frac{d I_{2}(G, \Gamma)}{d \mathcal{H}^{N-1}\left\lfloor S_{G}\right.}\left(x_{0}\right) \\
& \leqslant \limsup _{\delta \rightarrow 0^{+}} \limsup _{n \rightarrow+\infty} \frac{1}{\delta^{N-1}}\left[\int_{Q_{\nu}\left(x_{0}, \delta\right)} W\left(x, G(x), \nabla w_{n, \delta}(x)\right) d x\right.
\end{aligned}
$$




$$
\begin{gathered}
\left.+\int_{Q_{\nu}\left(x_{0}, \delta\right) \cap S_{w_{n}, \delta}} \Psi_{2}\left(x,\left[w_{n, \delta}(x)\right], \nu_{w_{n, \delta}}(x)\right) d \mathcal{H}^{N-1}(x)\right] \\
\leqslant \int_{Q_{\nu}} W^{\infty}\left(x_{0}, G\left(x_{0}\right), \nabla v(x)\right) d x+\int_{S_{v} \cap Q_{\nu}} \Psi_{2}\left(x_{0},[v(x)], \nu_{v}(x)\right) d \mathcal{H}^{N-1}(x)+O(\varepsilon),
\end{gathered}
$$

where we use hypotheses (H2) and (H6) to fix $x_{0}$ and $G\left(x_{0}\right)$, (H4) to pass from $W$ to $W^{\infty}$, and periodicity arguments. Thus, letting $\varepsilon \rightarrow 0^{+}$, the desired inequality follows by (5.32).

\section{EXAMPLE AND APPLiCATions}

6.1. An example. We provide an example in which the initial energy depends only on jumps in gradients through a specific initial interfacial energy $\Psi_{2}$ and in which an explicit formula for the bulk relaxed energy density emerges. Consider the initial energy $E$ in (3.1) with $W=0, \Psi_{1}=0$ and, for $a \in \mathbb{R}^{N}$ a fixed unit vector,

$$
\Psi_{2}(x, J, \nu)=|\nu \cdot J a|,
$$

for all $x \in \Omega, J \in \mathbb{R}^{N \times N}$, and $\nu \in S^{N-1}$.

From Theorem 3.2, and in view of Remark 3.1(4), we have that $W_{1}=0$, and we have the following cell formula for the bulk part $W_{1}+W_{2}=W_{2}$ of the relaxed energy in this setting: for almost every $x \in \Omega, A \in \mathbb{R}^{N \times N}, L, M \in \mathbb{R}^{N \times N \times N}$

$$
\begin{array}{r}
W_{2}(x, A, L, M)=\inf _{u \in S B V\left(Q ; \mathbb{R}^{N \times N}\right)}\left\{\int_{Q \cap S_{u}}\left|\nu_{u}(y) \cdot[u](y) a\right| d \mathcal{H}^{N-1}(y):\right. \\
\left.\left.u\right|_{\partial Q}(y)=L y, \int_{Q} \nabla u(y) d y=M\right\} .
\end{array}
$$

Consequently, $W_{2}$ does not depend upon $x$ and $A$, and we omit these variables. It is helpful in what follows to use the fact that each element $M \in \mathbb{R}^{N \times N \times N}$ can be identified with a bilinear mapping from $\mathbb{R}^{N}$ into $\mathbb{R}^{N}$

$$
\mathbb{R}^{N} \times \mathbb{R}^{N} \ni(y, z) \longmapsto M(y, z) \in \mathbb{R}^{N}
$$

where we have used the same symbol for the matrix and its associated bilinear mapping. Specifically, we may put

$$
M(y, z)_{i}=\sum_{j, k=1}^{N} M_{i j k} y_{j} z_{k} \text { for all } y, z \in \mathbb{R}^{N} .
$$

We denote the set of bilinear mappings on $\mathbb{R}^{N}$ with values in $\mathbb{R}^{N}$ by $\operatorname{Lin}^{2}\left(\mathbb{R}^{N}\right)$, and we note that for each $M \in \operatorname{Lin}^{2}\left(\mathbb{R}^{N}\right)$ the mapping $M(\cdot, a)$ is a linear mapping on $\mathbb{R}^{N}$ with values in $\mathbb{R}^{N}$, i.e., $M(\cdot, a) \in \operatorname{Lin}\left(\mathbb{R}^{N}\right)$.

Our main result here is the following explicit formula for $W_{2}$ in (6.2): for all $L, M \in$ $\operatorname{Lin}^{2}\left(\mathbb{R}^{N}\right)$

$$
W_{2}(L, M)=|\operatorname{tr}(L(\cdot, a)-M(\cdot, a))|
$$

where $\operatorname{tr}$ denotes the trace operation on $\operatorname{Lin}\left(\mathbb{R}^{N}\right)$. In terms of the associated elements of $\mathbb{R}^{N \times N \times N}$ the formula 6.4 reads

$$
W_{2}(L, M)=\left|\sum_{i, j=1}^{N}\left(L_{i i j}-M_{i i j}\right) a_{j}\right|
$$

With reference to Theorem 3.2 , when $W=0, \Psi_{1}=0$, and $\Psi_{2}$ is given by (6.1), we conclude that, for all $(g, G, \Gamma) \in S D^{2}\left(\Omega ; \mathbb{R}^{d}\right)$, the bulk part of the relaxed energy $I(g, G, \Gamma)$ is given by the integral

$$
\int_{\Omega} W_{2}(\nabla G(x), \Gamma(x)) d \mathcal{L}^{N}(x)=\int_{\Omega}|\operatorname{tr}((\nabla G(x)-\Gamma(x))(\cdot, a))| d \mathcal{L}^{N}(x) .
$$


This formula shows explicitly how the volume density of gradient disarrangements $\nabla G-$ $\Gamma$ determines the bulk relaxed energy associated with the purely interfacial initial energy density

$$
E(u)=\int_{S_{\nabla u} \cap \Omega}\left|\nu_{\nabla u} \cdot[\nabla u] a\right| d \mathcal{H}^{N-1} .
$$

It is worth noting that the initial energy density $E(u)$ measures the non-tangential part of the jumps in the directional derivative $(\nabla u) a$, so that the integrand in (6.6) provides for the second-order structured deformation $(g, G, \Gamma)$ an optimal volume density that accounts for non-tangential jumps in the directional derivative $(\nabla u) a$ of approximating deformations $u$.

To verify (6.4), we use Theorem 2 of [29] and follow the strategy in the proof of Lemma 2 in that article. As in their proof, a simple argument based on the triangle inequality and the Divergence Theorem for functions of bounded variation shows that $|\operatorname{tr}(L(\cdot, a)-M(\cdot, a))|$ is a lower bound for $W_{2}(L, M)$. To show the opposite inequality, we first consider the case in which the linear mapping $L(\cdot, a)-M(\cdot, a)$ is in the set $\mathcal{S} \subset \operatorname{Lin}\left(\mathbb{R}^{N}\right)$ of linear mappings with $N$ distinct eigenvalues each having non-zero real part and each with trace non-zero. According to Theorem 1 in [29], $\mathcal{S}$ is dense in $\operatorname{Lin}\left(\mathbb{R}^{N}\right)$. Let $R \subset Q$ be in the set $\mathcal{A}$ of all sets of finite perimeter having non-zero volume and compactly contained in $Q$. We define $u_{R}: Q \longrightarrow \mathbb{R}^{N \times N}$ by

$$
u_{R}(x)= \begin{cases}L x & \text { if } x \in Q \backslash R \\ |R|^{-1}(M-(1-|R|) L) x & \text { if } x \in R\end{cases}
$$

and note that $u_{R} \in S B V\left(Q, \mathbb{R}^{N \times N}\right)$, its jump set $S_{u_{R}}$ is included in $\partial^{*} R$ (the essential boundary of $R$, see [4]), and

$$
\left[u_{R}\right](x)=|R|^{-1}(L-M) x \quad \text { for } \mathcal{H}^{N-1} \text {-a.e. } x \in \partial R .
$$

These properties of $u_{R}$ and the arbitrariness of $R$ imply that for all $R \in \mathcal{A}$

$$
W_{2}(L, M) \leqslant|R|^{-1} \int_{\partial R}\left|\nu_{u_{R}}(x) \cdot((L-M) x) a\right| d \mathcal{H}^{N-1}(x)
$$

so that $W_{2}(L, M)$ does not exceed the infimum of the right-hand side with respect to $R \in \mathcal{A}$. Because $L(\cdot, a)-M(\cdot, a)$ is in the set $\mathcal{S}$ we may apply Theorem 2 of [29] to conclude

$$
W_{2}(L, M) \leqslant|\operatorname{tr}(L(\cdot, a)-M(\cdot, a))|
$$

which implies the equality (6.4) when $L(\cdot, a)-M(\cdot, a) \in \mathcal{S}$.

In order to verify (6.4) for arbitrary $L, M \in \operatorname{Lin}^{2}\left(\mathbb{R}^{N}\right)$, we first note that for each $z \in \mathbb{R}^{N}$ we may write $z=(z \cdot a) a+z_{\perp}$ where $z_{\perp} \cdot a=0$. Now put $\Delta=L-M$ and notice that, by the linearity of $\Delta(y, \cdot)$, there holds

$$
\begin{aligned}
\Delta(y, z) & =\Delta\left(y,(z \cdot a) a+z_{\perp}\right) \\
& =(z \cdot a) \Delta(y, a)+\Delta\left(y, z_{\perp}\right) .
\end{aligned}
$$

Since $\Delta(\cdot, a) \in \operatorname{Lin}\left(\mathbb{R}^{N}\right)$ and $\mathcal{S}$ is dense in $\operatorname{Lin}\left(\mathbb{R}^{N}\right)$, we may choose a sequence $n \longmapsto A_{n} \in \mathcal{S}$ such that $\lim _{n \rightarrow \infty} A_{n}=\Delta(\cdot, a)$. We set

$$
\Delta_{n}(y, z)=(z \cdot a) A_{n} y+\Delta\left(y, z_{\perp}\right)
$$

and observe that $\Delta_{n} \in \operatorname{Lin}^{2}\left(\mathbb{R}^{N}\right)$ and for all $y, z \in \mathbb{R}^{N}$

$$
\begin{aligned}
\lim _{n \rightarrow \infty} \Delta_{n}(y, z) & =(z \cdot a) \Delta(y, a)+\Delta\left(y, z_{\perp}\right)=\Delta(y, z) \\
& =(L-M)(y, z) .
\end{aligned}
$$

Putting $M_{n}=L-\Delta_{n}$, we conclude that $\lim _{n \rightarrow \infty} M_{n}=L-\lim _{n \rightarrow \infty} \Delta_{n}=M$ as well as

$$
\begin{aligned}
\left(L-M_{n}\right)(y, a) & =\Delta_{n}(y, a) \\
& =(a \cdot a) A_{n} y+\Delta\left(y, a_{\perp}\right)=A_{n} y,
\end{aligned}
$$

so that $\left(L-M_{n}\right)(\cdot, a) \in \mathcal{S}$. (In the last step we have used the fact that $a_{\perp}=0$.) Therefore, $W_{2}\left(L, M_{n}\right)=\left|\operatorname{tr} A_{n}\right|$, and letting $n \rightarrow \infty$ and using the continuity of $W_{2}(L, \cdot)$ established in Proposition 4.11 and of the trace operator we conclude that 


$$
\begin{aligned}
W_{2}(L, M) & =\lim _{n \rightarrow \infty} W_{2}\left(L, M_{n}\right)=\lim _{n \rightarrow \infty}\left|\operatorname{tr} A_{n}\right|=\left|\operatorname{tr} \lim _{n \rightarrow \infty} A_{n}\right| \\
& =|\operatorname{tr}(L-M)(\cdot, a)|
\end{aligned}
$$

and thereby complete the verification of (6.4).

6.2. Applications. For the case $\Omega \subset \mathbb{R}^{3}$ the relaxed energies for first-order structured deformations $(g, G) \in S B V^{2}\left(\Omega, \mathbb{R}^{3}\right) \times S B V\left(\Omega, \mathbb{R}^{3 \times 3}\right)$ studied in [7] provide a means of capturing the effect of both submacroscopically smooth changes and of submacroscopically non-smooth geometrical changes (disarrangements) on the bulk energy stored in a three-dimensional body. In particular, the bulk relaxed energy density $(A, B) \longmapsto W_{1}(A-B)$ of [7] provides the portion

$$
I_{d i s}(g, G)=\int_{\Omega} W_{1}(\nabla g(x)-G(x)) d \mathcal{L}^{3}(x)
$$

of the bulk part of the relaxed energy that arises from disarrangements. This interpretation of $I_{d i s}(g, G)$ is justified by considering a sequence $\left\{u_{n}\right\}$ in $S B V^{2}\left(\Omega, \mathbb{R}^{3}\right)$ with $u_{n} \rightarrow g$ and $\nabla u_{n} \rightarrow G$ both in $L^{1}$ and by writing

$$
\begin{aligned}
\nabla g \mathcal{L}^{3}+[g] \otimes \nu_{g} \mathcal{H}^{2} & =D g=D \lim _{n \rightarrow \infty} u_{n}=\lim _{n \rightarrow \infty} D u_{n} \\
& =\lim _{n \rightarrow \infty}\left(\nabla u_{n} \mathcal{L}^{3}+\left[u_{n}\right] \otimes \nu_{u_{n}} \mathcal{H}^{2}\right) \\
& =G \mathcal{L}^{3}+\lim _{n \rightarrow \infty}\left(\left[u_{n}\right] \otimes \nu_{u_{n}} \mathcal{H}^{2}\right)
\end{aligned}
$$

showing that $M \mathcal{L}^{3}:=(\nabla g-G) \mathcal{L}^{3}$ is the absolutely continuous part of the limit of the singular measures $\left[u_{n}\right] \otimes \nu_{u_{n}} \mathcal{H}^{2}$ that capture the submacroscopic disarrangements associated with $(g, G)$. Moreover, the energy density $(A, L) \longmapsto W_{2}(A, L)$ of [7] provides the remaining portion

$$
I \backslash(g, G)=\int_{\Omega} W_{2}(G(x), \nabla G(x)) d \mathcal{L}^{3}(x)
$$

of the bulk part of the relaxed energy, namely, the portion that arises without disarrangements.

The availability in [7] (or, alternatively, directly from the results of [12]) of such refined bulk energies provides connections to the research [17] that attempts to broaden classical, finite elasticity into the setting of first-order structured deformations through the field theory "elasticity with disarrangements". (That theory requires the specification at the outset of a bulk energy in the form $\int_{\Omega} \Psi(G(x), \nabla g(x)) d \mathcal{L}^{3}(x)$, so that, for applications of energy relaxation to elasticity with disarrangements, the dependence of the bulk density $W_{2}$ on the third-order tensor field $\nabla G$ in the formula for $I \backslash(g, G)$ can be dropped). Elasticity with disarrangements [17] has been applied to the study of granular materials [18, [19, 20], with $G$ representing the smooth deformation of grains and with $g$ representing the macroscopic deformation of the aggregate of grains, and this broadened version of finite elasticity has provided a setting in which no-tension materials with non-linear response in compression arise in a natural way.

While the scope of elasticity with disarrangements is broad enough to capture some energetic effects of disarrangements, its setting in the context of first-order structured deformations precludes its capturing directly the effects of "gradient disarrangements", i.e., of jumps in the gradients of deformations that approximate geometrical changes at the smaller length scale. The theory of second-order structured deformations $(g, G, \Gamma) \in S B V^{2}\left(\Omega, \mathbb{R}^{3}\right) \times$ $S B V\left(\Omega, \mathbb{R}^{3 \times 3}\right) \times L^{1}\left(\Omega, \mathbb{R}^{3 \times 3 \times 3}\right)$ guarantees the existence of a sequence $n \longmapsto u_{n} \in S B V^{2}\left(\Omega, \mathbb{R}^{3}\right)$ such that $u_{n} \rightarrow g$ and $\nabla u_{n} \rightarrow G$ in $L^{1}$ while $\nabla^{2} u_{n}$ tends to $\Gamma$ weakly in the sense of measures. 
Following the idea of the calculation $(6.9)$ we have

$$
\begin{aligned}
\nabla^{2} g \mathcal{L}^{3}+[\nabla g] \otimes \nu_{\nabla g} \mathcal{H}^{2}= & D \nabla g \\
= & D(\nabla g-G)+D G=D M+D \lim _{n \rightarrow \infty} \nabla u_{n} \\
= & D M+\lim _{n \rightarrow \infty} D \nabla u_{n} \\
= & \nabla M \mathcal{L}^{3}+[M] \otimes \nu_{[M]} \mathcal{H}^{2} \\
& +\lim _{n \rightarrow \infty}\left(\nabla^{2} u_{n} \mathcal{L}^{3}+\left[\nabla u_{n}\right] \otimes \nu_{\nabla u_{n}} \mathcal{H}^{2}\right) \\
= & (\nabla M+\Gamma) \mathcal{L}^{3}+[M] \otimes \nu_{[M]} \mathcal{H}^{2} \\
& +\lim _{n \rightarrow \infty}\left(\left[\nabla u_{n}\right] \otimes \nu_{\nabla u_{n}} \mathcal{H}^{2}\right),
\end{aligned}
$$

which shows that $\nabla^{2} g-\nabla M-\Gamma=\nabla^{2} g-\nabla(\nabla g-G)-\Gamma=\nabla G-\Gamma$ is the absolutely continuous part of the distributional limit as $n \rightarrow \infty$ of the singular measures $\left[\nabla u_{n}\right] \otimes \nu_{\nabla u_{n}} \mathcal{H}^{2}$.

We conclude that each second-order structured deformation $(g, G, \Gamma)$ provides the field $\nabla G-$ $\Gamma \in L^{1}\left(\Omega, \mathbb{R}^{3 \times 3 \times 3}\right)$ that serves as a volume density of gradient disarrangements. Moreover, since the initial pair $(g, G)$ in the triple $(g, G, \Gamma)$ is a first-order structured structured deformation, the field $\nabla g-G \in S B V\left(\Omega, \mathbb{R}^{3 \times 3}\right)$ remains available as a volume density of disarrangements. Consequently, the results in the present paper on relaxation in the context of second-order structured deformations capture the influence both of disarrangements and of gradient-disarrangements on relaxed energies and provide the starting point for broadening elasticity with disarrangements to the richer multiscale geometry of second-order structured deformations. Initial steps toward such a broadening have been taken [27] in the context of second-order structured deformations. A physical context of significance - phasetransitions in metals [1, 2, 24] - provides a setting in which deformations can be approximately piecewise homogeneous at small length scales. In this setting it is appropriate to assume that there are approximating piecewise smooth deformations $u_{n}$ with the property $\Gamma=\lim _{n \rightarrow \infty} \nabla^{2} u_{n}=0$. Second-order structured deformations of the form $(g, G, 0)$ are called submacroscopically affine, and, for them, the gradient-disarrangement density $\nabla G-\Gamma$ reduces to $\nabla G$, i.e., the "strain-gradient" quantity $\nabla G$ measures the volume density of jumps in gradients of approximating piecewise affine deformations. The results of the present paper provide in particular an energetics of bodies undergoing submacroscopically affine structured deformations and, looking ahead, will provide the constitutive input for the field theory "elasticity with gradient disarrangements" applied to bodies undergoing deformations that are approximately piecewise homogeneous at small length scales.

Acknowledgements. The authors warmly thank the Center for Nonlinear Analysis (NSF Grants No. DMS-0405343 and DMS-0635983) at Carnegie Mellon University, Pittsburgh, USA, CAMGSD at Instituto Superior Técnico, Lisbon, Portugal, and SISSA, Trieste, Italy, where this research was carried out. MM is a member of the Progetto di Ricerca GNAMPAINdAM 2015 "Fenomeni critici nella meccanica dei materiali: un approccio variazionale". The research of ACB, JM, and MM was partially supported by the Fundação para a Ciência e a Tecnologia (Portuguese Foundation for Science and Technology) through the CMU-Portugal Program under grant FCT-UTA_CMU/MAT/0005/2009 "Thin Structures, Homogenization, and Multiphase Problems". The research of ACB was partially supported by the Fundação para a Ciência e a Tecnologia through grant UID/MAT/04561/2013. The research of MM was partially supported by the European Research Council through the ERC Advanced Grant "QuaDynEvoPro", grant agreement no. 290888. The research of JM was partially supported by the Fundação para a Ciência e a Tecnologia through grant UID/MAT/04459/2013.

\section{REFERENCES}

[1] A. ACharya And C. Fressengeas: Continuum mechanics of the interaction of phase boundaries and dislocations in solids. In Differential Geometry and Continuum Mechanics, Ed: G. Q Chen, M. Grinfeld, R.J. Knops, Springer Proceedings in Mathematics \& Statistics 137 (2015), 125-168. 
[2] V. Agrawal AND K. Dayal: Dynamic Phase-field Model for Structural Transformations and Twinning: Regularized Interfaces with Transparent Prescription of Complex Kinetics and Nucleation. Part I: Formulation and One-Dimensional Characterization. J. Mech. Phys. Solids 85 (2015), 270-290.

[3] G. Alberti: A Lusin type theorem for gradients, J. Funct. Anal. 100 (1991), 110-118.

[4] L. Ambrosio, N. Fusco, AND D. Pallara: Functions of Bounded Variation and Free Discontinuity Problems, Oxford University Press, 2000.

[5] L. Ambrosio, S. Mortola, And V. M. Tortorelli: Functionals with linear growth defined on vector valued $B V$ functions, J. Math. Pures et Appl. 70 (1991), 269-323.

[6] M. BAÍA, J. MATiAs, AND P. M. SANTos: A survey of structured deformations, São Paulo Journal of Mathematical Sciences, 5 (2011), 185-201.

[7] M. Baía, J. Matias, AND P. M. SANTos: A relaxation result in the framework of structured deformations, Proc. Royal Soc. Edinburgh Sect. A 142A (2012), 239-271.

[8] S. BALDO: Minimal interface criterion for phase transitions in mixtures of Cahn-Hilliard fluids, Ann. Inst. Henri Poincaré. Anal. Non Linéaire 7 No.2 (1990), 67-90.

[9] A. C. Barroso, G. Bouchitté, G. Buttazzo, And I. Fonseca: Relaxation of bulk and interfacial energies, Arch. Rational Mech. Anal. 135 (1996), 107-173.

[10] M. Carriero, A. Leaci, AND F. Tomarelli: A second order model in image segmentation: Blake and Zisserman Functional, 25, Progress in Nonlinear Diff. Equations, (1996), 57-72.

[11] M. Carriero, A. Leaci, AND F. Tomarelli: Second order variational problems with free discontinuity and free gradient discontinuity, Calculus of Variations: Topics from the Mathematical Heritage of E. De Giorgi, Quad. Mat., 14, pp. 135-186. Dept. Math., Seconda Univ. Napoli, Caserta (2004).

[12] R. CHOKSI AND I. FONSECA: Bulk and interfacial energies for structured deformations of continua, Arch. Rational Mech. Anal. 138 (1997), 37-103.

[13] E. De Giorgi And L. Ambrosio: Un nuovo tipo di funzionale del calcolo delle variazioni, Atti Accad. Naz. Lincei, 82 (1988), 199-210.

[14] G. DeL Piero: The energy of a one-dimensional structured deformation, Math. Mech. Solids 6 (2001), 387-408.

[15] G. Del Piero AND D. R. Owen: Structured deformations of continua, Arch. Rational Mech. Anal. 124 (1993), 99-155.

[16] G. Del Piero And D. R. Owen: Multiscale Modeling in Continuum Mechanics and Structured Deformations. CISM Courses and Lecture Notes 447, (2004) Springer-Verlag.

[17] L. DESERI AND D. R. Owen: Toward a field theory for elastic bodies undergoing disarrangements. J. Elas. 70 (2003), 197-236.

[18] L. DESERI AND D. R. OWEN: Moving interfaces that separate loose and compact phases of elastic aggregates: a mechanism for drastic reduction or increase in macroscopic deformation. Cont. Mech. Thermo. 25 (2013), 311-341.

[19] L. DESERI AND D. R. OWEN: Stable disarrangement phases of elastic aggregates: a setting for the emergence of no-tension materials with non-linear response in compression. Meccanica 49 (2014), 2907-2932.

[20] L. DESERI AND D. R. OWEN: Stable disarrangement phases arising from expansion/contraction or from simple shearing of a model granular medium. Int. J. Eng. Sci. 96 (2015), 111-140.

[21] L. C. Evans AND R. F. GARIEPY: Measure Theory and Fine Properties of Functions, Studies in Advanced Mathematics, CRC Press, 1992.

[22] H. FEDERER: Geometric Measure Theory, Springer, Berlin, 1969.

[23] E. Giusti: Minimal Surfaces and Functions of Bounded Variation, Birkhäuser, 1984.

[24] R. D. JAMES AND K. F. HANE: Martensitic transformations and shape-memorymaterials. Acta Mater. 48 (2000), 197-222.

[25] C. J. LARSEN: On the representation of effective energy densities, ESAIM: Control, Opt., Calc. Var. 5 (2000), 529-538.

[26] J. MATIAS: Differential inclusions in $S B V_{0}(\Omega)$ and applications to the Calculus of Variations, J. of Convex Analysis 14 (2007), No. 3, 465-477.

[27] D. R. Owen: Elasticity with Gradient-Disarrangements: a Multiscale Geometrical Perspective for StrainGradient Theories of Elasticty and of Plasticity. J. Elasticity submitted.

[28] D. R. OWEn AND R. PARONI: Second-order structured deformations, Arch. Rational Mech. Anal. 155 (2000), 215-235.

[29] D. R. OWEN AND R. PARONI: Optimal flux densities for linear mappings and the multiscale geometry of structured deformations, Arch. Ration. Mech. Anal. 218 (2015), no. 3, 1633-1652.

[30] R. PARONI: Second-order structured deformations: approximation theorems and energetics, in: Multiscale modeling in continuum mechanics and structured deformations, edited by G. Del Piero and D. R. Owen, CISM 447, Springer (2004).

[31] Y. G. RESHETNYAK: Weak convergence of completely additive vector functions on a set. Siberian Math. J. 9 (1968), 1039-1045 (translation of Sibirsk Mat. Z. 9 (1968), 1386-1394.

[32] M. ŠILHAVÝ: On the approximation theorem for structured deformations from $B V(\Omega)$, Mech. Math. Complex. Syst. 3, (2015), 83-100.

[33] W. Ziemer: Weakly Differentiable Functions. Springer-Verlag, 1989. 
SECOND-ORDER STRUCTURED DEFORMATIONS: RELAXATION, INTEGRAL REPRESENTATION AND APPLICATION\$5

Faculdade de Ciências da Universidade de lisboa, Departamento de Matemática and CMaF-CiO, Campo Grande, Edifício C6, Piso 1, 1749-016 Lisboa, Portugal

E-mail address, A. C. Barroso: acbarroso@ciencias.ulisboa.pt

Departamento de Matemática, Instituto Superior Técnico, Av.Rovisco Pais, 1, 1049-001 Lisboa, PORTUGAL

E-mail address, J. Matias: jose.c.matias@tecnico.ulisboa.pt

SisSA - International School for Advanced Studies, Via Bonomea, 265, 34136 Trieste, Italy

E-mail address, M. Morandotti ( $\square)$ : marco.morandotti@sissa.it

Department of Mathematical Sciences, Carnegie Mellon University, 5000 Forbes Ave., PittsBURGH, PA 15213 USA

E-mail address, D. R. Owen: do 04 @andrew.cmu.edu 